\title{
Local Number Fluctuations in Hyperuniform and Nonhyperuniform Systems: Higher-Order Moments and Distribution Functions
}

\author{
Salvatore Torquato $\odot,{ }^{1,2, *}$ Jaeuk Kim $\odot,{ }^{1, \dagger}$ and Michael A. Klatt $\oplus^{1,3, *}$ \\ ${ }^{1}$ Department of Physics, Princeton University, Princeton, New Jersey 08544, USA \\ ${ }^{2}$ Department of Chemistry, Princeton Institute for the Science and Technology of Materials, \\ and Program in Applied and Computational Mathematics, Princeton University, \\ Princeton, New Jersey 08544, USA \\ ${ }^{3}$ Institut für Theoretische Physik, FAU Erlangen-Nürnberg, \\ Staudtstr. 7, 91058 Erlangen, Germany
}

(Received 10 December 2020; revised 26 February 2021; accepted 3 March 2021; published 5 May 2021)

The local number variance $\sigma^{2}(R)$ associated with a spherical sampling window of radius $R$ enables a classification of many-particle systems in $d$-dimensional Euclidean space $\mathbb{R}^{d}$ according to the degree to which large-scale density fluctuations are suppressed, resulting in a demarcation between hyperuniform and nonhyperuniform phyla. To more completely characterize density fluctuations, we carry out an extensive study of higher-order moments or cumulants, including the skewness $\gamma_{1}(R)$, excess kurtosis $\gamma_{2}(R)$, and the corresponding probability distribution function $P[N(R)]$ of a large family of models across the first three space dimensions, including both hyperuniform and nonhyperuniform systems with varying degrees of short- and long-range order. To carry out this comprehensive program, we derive new theoretical results that apply to general point processes, and we conduct high-precision numerical studies. Specifically, we derive explicit closed-form integral expressions for $\gamma_{1}(R)$ and $\gamma_{2}(R)$ that encode structural information up to three-body and four-body correlation functions, respectively. We also derive rigorous bounds on $\gamma_{1}(R), \gamma_{2}(R)$, and $P[N(R)]$ for general point processes and corresponding exact results for general packings of identical spheres. High-quality simulation data for $\gamma_{1}(R), \gamma_{2}(R)$, and $P[N(R)]$ are generated for each model. We also ascertain the proximity of $P[N(R)]$ to the normal distribution via a novel Gaussian "distance" metric $l_{2}(R)$. Among all models, the convergence to a central limit theorem (CLT) is generally fastest for the disordered hyperuniform processes in two or higher dimensions such that $\gamma_{1}(R) \sim l_{2}(R) \sim$ $R^{-(d+1) / 2}$ and $\gamma_{2}(R) \sim R^{-(d+1)}$ for large $R$. The convergence to a CLT is slower for standard nonhyperuniform models and slowest for the "antihyperuniform" model studied here. We prove that one-dimensional hyperuniform systems of class I or any $d$-dimensional lattice cannot obey a CLT. Remarkably, we discover a type of universality in that, for all of our models that obey a CLT, the gamma distribution provides a good approximation to $P[N(R)]$ across all dimensions for intermediate to large values of $R$, enabling us to estimate the large- $R$ scalings of $\gamma_{1}(R), \gamma_{2}(R)$, and $l_{2}(R)$. For any $d$-dimensional model that "decorrelates" or "correlates" with $d$, we elucidate why $P[N(R)]$ increasingly moves toward or away from Gaussian-like behavior, respectively. Our work sheds light on the fundamental importance of higher-order structural information to fully characterize density fluctuations in many-body systems across length scales and dimensions, and thus has broad implications for condensed matter physics, engineering, mathematics, and biology.

DOI: $10.1103 /$ PhysRevX.11.021028

Subject Areas: Condensed Matter Physics

Soft Matter, Statistical Physics

\footnotetext{
*torquato@princeton.edu

jaeukk@princeton.edu

${ }^{\ddagger}$ mklatt@ princeton.edu
}

Published by the American Physical Society under the terms of the Creative Commons Attribution 4.0 International license. Further distribution of this work must maintain attribution to the author(s) and the published article's title, journal citation, and DOI. 


\section{INTRODUCTION}

The quantification of density fluctuations in manyparticle systems in $d$-dimensional Euclidean space $\mathbb{R}^{d}$ is of great fundamental and practical importance in many fields across the physical, mathematical, and biological sciences [1-16]. It is well known that long-wavelength density fluctuations of disordered as well as ordered systems contain crucial information about the structure as well as equilibrium and nonequilibrium physical properties of the systems $[1,5,13,16]$. Clearly, density fluctuations that occur on some arbitrary local length scale $[2,3,8,9$, 11-13,15-18] provide considerably more information about the system than those in the long-wavelength limit, as elaborated below.

Consider a statistically homogeneous (translationally invariant) point process in $d$-dimensional Euclidean space $\mathbb{R}^{d}$ at number density $\rho$ and sampling for the number of points $N(R)$ within a $d$-dimensional spherical window of radius $R$ (see Fig. 1) and volume

$$
v_{1}(R)=\frac{\pi^{d / 2} R^{d}}{\Gamma(1+d / 2)} .
$$

The local number variance $\sigma^{2}(R) \equiv\left\langle N^{2}(R)\right\rangle-\langle N(R)\rangle^{2}$ is a useful measure of number fluctuations, where the first moment $\langle N(R)\rangle=\rho v_{1}(R)$ is the average number of points within a $d$-dimensional spherical (sampling) window of radius $R$ and angular brackets denote an ensemble average. The local number variance is exactly determined by pair statistics and can be given either in terms of the pair

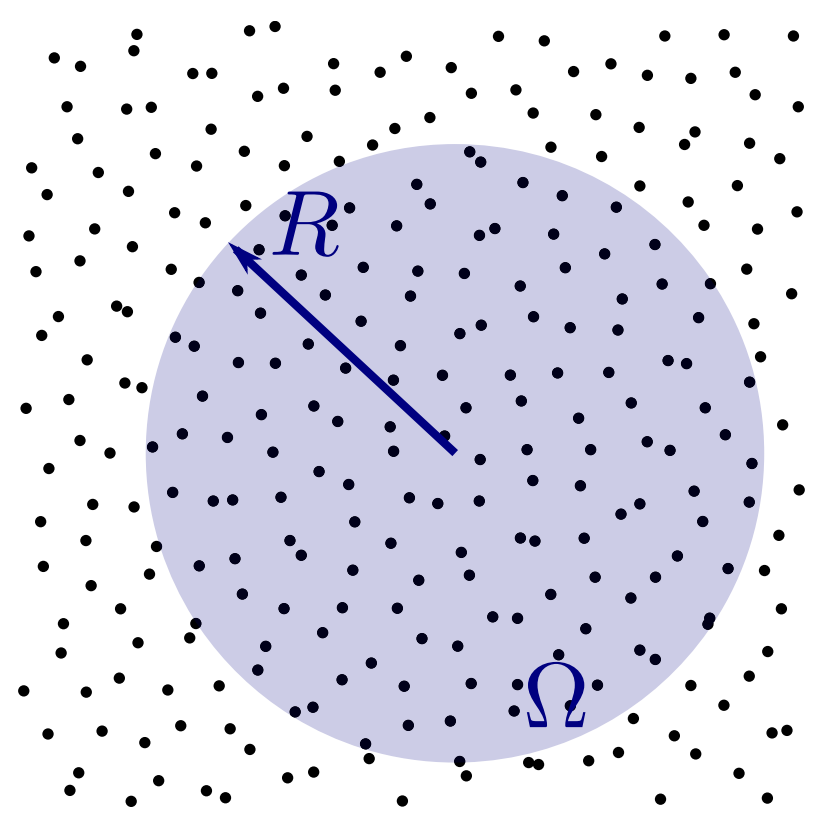

FIG. 1. Schematic showing a spherical window $\Omega$ of radius $R$ used to sample fluctuations in the number of points in a twodimensional point process. correlation function $g_{2}(\mathbf{r})$ in direct space or the structure factor $S(\mathbf{k})$ in reciprocal space [13]:

$$
\begin{aligned}
\sigma^{2}(R) & =\rho v_{1}(R)\left[1+\rho \int_{\mathbb{R}^{d}} h(\mathbf{r}) \alpha_{2}(r ; R) d \mathbf{r}\right], \\
& =\rho v_{1}(R)\left[\frac{1}{(2 \pi)^{d}} \int_{\mathbb{R}^{d}} S(\mathbf{k}) \tilde{\alpha}_{2}(k ; R) d \mathbf{k}\right],
\end{aligned}
$$

where $h(\mathbf{r}) \equiv g_{2}(\mathbf{r})-1$ is the total correlation function, $\alpha_{2}(r ; R)$ is the intersection volume of two spherical windows of radius $R$, scaled by $v_{1}(R)$, whose centers are separated by the distance $r$, and $\tilde{\alpha}_{2}(k ; R)$ is its Fourier transform. The large-scale behavior of the number variance $\sigma^{2}(R)$ is central to the hyperuniformity concept, which is attracting attention across many fields [13,16,19-23]. Specifically, a hyperuniform point process is one in which $\sigma^{2}(R)$ grows more slowly than the window volume, i.e., $R^{d}$, for large $R$ and hence is characterized by large-scale density fluctuations that are anomalously suppressed compared to those of typical disordered systems. The hyperuniformity concept generalizes the traditional notion of long-range order of crystals and quasicrystals to also encompass certain exotic disordered states of matter [13,16]. Disordered hyperuniform systems are diametrically opposed to systems at thermal critical points (e.g., liquid-vapor or magnetic critical points) in which the local variance diverges faster than $R^{d}$ in the limit $R \rightarrow \infty$. Any system with such divergent behavior in the local variance is called antihyperuniform [16] (see also Sec. II for additional details).

Our ensuing analysis to characterize fluctuations applies to hyperuniform and nonhyperuniform many-particle systems, which constitute all possible statistically homogeneous states of matter in any space dimension. Generally, such states of matter encompass crystals, crystals with defects, quasicrystals and their defective variants, typical gases, liquids and glasses, and exotic amorphous hyperuniform systems. Real-world materials and other example systems include atomic and molecular systems, particulate composites, colloids, polymers, trees in a forest, biological tissue, galaxy distributions, and plasmas, to mention but a few.

While the local number variance contains useful information, we would like to more completely characterize the fluctuations by ascertaining higher-order moments, i.e., $\left\langle N^{k}(R)\right\rangle$, where $k \geq 3$, as well as the corresponding discrete probability distribution $P[N(R)]$ associated with finding exactly $N(R)$ particles within a $d$-dimensional spherical window of radius $R$. The $m$ th moment of the distribution is given by

$$
\left\langle N^{m}(R)\right\rangle=\sum_{N(R)=0} N^{m}(R) P[N(R)]
$$

Because of the fact that the random variable $N(R)$ is discrete and cannot take on negative values, the probability 
distribution $P[N(R)]$, for finite $R$, can never exactly attain the normal distribution, which is given by

$$
P[N(R)]=\frac{1}{\sqrt{2 \pi} \sigma(R)} \exp \left[\frac{(N(R)-\langle N(R)\rangle)^{2}}{2 \sigma^{2}(R)}\right],
$$

where $\sigma(R)=\left[\sigma^{2}(R)\right]^{1 / 2}$ is the standard deviation of $N(R)$. For example, for a statistically homogeneous Poisson point process in $\mathbb{R}^{d}$ at number density $\rho$,

$$
P[N(R)]=\frac{\left[\rho v_{1}(R)\right]^{N(R)}}{N(R) !} \exp \left[-\rho v_{1}(R)\right],
$$

which deviates significantly from the normal distribution for sufficiently small $R$. This is one of the rare cases in which a closed-form analytic formula for $P[N(R)]$ is known across dimensions for nontrivial point processes. It is only when $R$ tends to infinity that the Poisson distribution becomes a normal distribution, i.e., it follows a central limit theorem (CLT); see Ref. [24] and references therein. The reader is referred to Refs. [25-28] for proofs of CLTs for other point processes.

There are many open fundamental and practical questions that we aim to address in this paper:

(i) What information is encoded in the higher-order moments of the number distribution that is not contained in the first two moments, i.e., number density and variance?

(ii) What hyperuniform and nonhyperuniform systems obey or disobey a CLT?

(iii) For those systems that obey a CLT, how large must $R$ be for $P[N(R)]$ to be well approximated by a Gaussian?

(iv) What are robust measures of non-Gaussianity?

(v) Can one derive exact results or bounds for the higher-order moments and $P[N(R)]$ ?

(vi) Can we theoretically predict the asymptotic behavior of the moments using well-known, closed-form probability distributions that approximate the actual distributions for finite $R$ ?

(vii) Do nonhyperuniform fluctuations behave differently from hyperuniform ones?

(viii) What is the effect of increasing the space dimension on number fluctuations for any particular model? How is this effect related to the correlations between particles?

In this paper, we show that higher-order moments of $N(R)$ and the full distribution function $P[N(R)]$ enable one to infer the importance of crucial higher-body correlation functions at various length scales, since these moments of $N(R)$ can be expressed, as shown later, as integrals over $n$ body correlation functions. This approach has very important implications for the physics of the systems since their physical properties are generally determined by the $n$-body correlation functions [5,29-33]. Thus, one can quantify the degree of the non-Gaussianity of the structure of a system, i.e., when the first two moments, determined by the density and pair correlation function, are insufficient to characterize the system. An outstanding example of inferring the physical processes that lead to a state of the system from the determination of the degree of non-Gaussianity in a system is the characterization of temperature fluctuations of the cosmic microwave background (CMB) [7]. In our work, we have laid the theoretical foundation to be able to study the structure of matter that would similarly enable one to infer the underlying physical processes that lead to them.

Here, we investigate the skewness $\gamma_{1}(R)$ (related to the first three moments), excess kurtosis $\gamma_{2}(R)$ (related to the first four moments), and the number distribution function $P[N(R)]$ for general homogeneous point processes in $\mathbb{R}^{d}$, as well as a wide class of models across dimensions. Specifically, we derive explicit closed-form integral expressions for $\gamma_{1}(R)$ in terms of the number density $\rho$, the pair correlation function $g_{2}$, and the three-body correlation function $g_{3}$ (defined in Sec. II). Similarly, we derive the corresponding formulas for the excess kurtosis $\gamma_{2}(R)$, which now depend additionally on the four-body correlation function $g_{4}$. These integral relations also involve geometrical information about the spherical windows via the intersection volumes of up to three and four spheres in the cases of the skewness and excess kurtosis, respectively. Thus, the skewness and excess kurtosis encode up to threebody and four-body information about spatial correlations and window geometries, respectively. We also derive some exact elementary results for the skewness, excess kurtosis, and number distribution that apply to general packings of identical spheres. Importantly, such rigorous bounds can be applied to check the corresponding results obtained from both numerical and experimental studies.

Via high-precision computer simulation studies, we accurately determine the number variance, skewness, excess kurtosis, and the number distribution for up to eight different models of statistically homogeneous point processes across the first three space dimensions and a wide range of window radii $R$. These models, summarized in Table I, include both nonhyperuniform and hyperuniform systems with varying degrees of short- and long-range order. Importantly, we choose our models so that they span a representative subset of all possible ranges of $S(0)$, including stealthy hyperuniform systems, nonstealthy hyperuniform ones, nearly hyperuniform systems, and those with $S(0)$ of order unity (gases) or larger, i.e., super-Poissonian systems as well as the most extreme nonhyperuniform cases (antihyperuniform). These choices were made so that they are representative of other physical systems (see Table II) with similar values of $S(0)$. Among the nonhyperuniform point processes, we characterize fluctuations of Poisson, random sequential addition (RSA) packings, equilibrium hard spheres, 
TABLE I. Simulation parameters for all of the model point processes across the first three space dimensions considered in this work. Here, $\mathcal{N}$ is the average number of points inside a fundamental cell, $N_{c}$ is the number of point patterns considered, $N_{\text {window }}$ is the number of observation windows per point pattern, and $R_{\max }$ is the largest radius of an observation window. We have also indicated the values of the structure factors at the origin, $S(0)$. In the case of equilibrium packings, the values of $S(0)$ are obtained from the exact result for hard rods $(d=1)$ [34] and highly accurate approximations for the conditional nearest-neighbor function $G_{P}(r)$ in the limit $r \rightarrow \infty$ [35,36]. Note that our computer simulation results are in excellent agreement with these analytical estimates of $S(0)$ for equilibrium packings.

\begin{tabular}{|c|c|c|c|c|c|c|}
\hline Models & & $\mathcal{N}$ & $N_{c}$ & $N_{\text {window }}$ & $R_{\max } \rho^{1 / d}$ & $S(0)$ \\
\hline \multirow[t]{3}{*}{ Antihyperuniform } & 2D HIP & $\infty$ & $10^{6}$ & 1 & 50 & $\infty$ \\
\hline & 3D HIP & $\infty$ & $10^{6}$ & 1 & 30.1 & $\infty$ \\
\hline & 4D HIP & $\infty$ & $10^{4}$ & 1 & 5.4 & $\infty$ \\
\hline \multirow[t]{12}{*}{ Standard Nonhyperuniform } & 1D Poisson cluster & 105 & $10^{6}$ & 1 & 50 & 11 \\
\hline & 2D Poisson cluster & $205^{2}$ & $10^{7}$ & 1 & 100 & 11 \\
\hline & 3D Poisson cluster & $45^{3}$ & $10^{7}$ & 1 & 20 & 11 \\
\hline & 1D Poisson [37] & $\infty$ & 1 & $\infty$ & 50 & 1 \\
\hline & 2D Poisson [37] & $\infty$ & 1 & $\infty$ & 50 & 1 \\
\hline & 3D Poisson [37] & $\infty$ & 1 & $\infty$ & 50 & 1 \\
\hline & $1 \mathrm{D} \operatorname{RSA}(\phi=0.74)$ & $10^{7}$ & 9987 & $10^{2}$ & 50 & $0.051[38]$ \\
\hline & $2 \mathrm{D} \operatorname{RSA}(\phi=0.55)$ & $10^{4}$ & $10^{4}$ & $10^{3}$ & 25 & $0.05869(4)[39]$ \\
\hline & $3 \mathrm{D} \operatorname{RSA}(\phi=0.38)$ & $10^{6}$ & 250 & $10^{4}$ & 25 & $0.05581(5)[39]$ \\
\hline & 1D Equil. hard rods $(\phi=0.75)$ & $5 \times 10^{3}$ & $10^{3}$ & 10 & 100 & $0.0629(2)$ \\
\hline & 2D Equil. hard disks $(\phi=0.65)$ & $10^{4}$ & $10^{3}$ & 10 & 15 & $0.0260(4)$ \\
\hline & 3D Equil. hard spheres $(\phi=0.48)$ & $10^{4}$ & 100 & $10^{3}$ & 20 & $0.022(1)$ \\
\hline \multirow[t]{9}{*}{ Hyperuniform } & 1D Cloaked URL & $10^{4}$ & $10^{6}$ & 1 & 50 & 0 \\
\hline & 2D Cloaked URL & $10^{4}$ & $10^{7}$ & 1 & 50 & 0 \\
\hline & 3D Cloaked URL & $44^{3}$ & $10^{7}$ & 1 & 20 & 0 \\
\hline & 1D Stealthy $(\chi=0.30)$ & $10^{3}$ & 900 & $10^{3}$ & 50 & 0 \\
\hline & 2D Stealthy $(\chi=0.49)$ & $10^{4}$ & 700 & $10^{2}$ & 50 & 0 \\
\hline & 3D Stealthy $(\chi=0.49)$ & $8 \times 10^{3}$ & $10^{3}$ & $10^{3}$ & 8 & 0 \\
\hline & Integer lattice [40] & $\infty$ & 1 & $\infty$ & 50 & 0 \\
\hline & Square lattice & $10^{4}$ & 1 & $10^{5}$ & 50 & 0 \\
\hline & SC lattice & $5.12 \times 10^{5}$ & 1 & $10^{4}$ & 40 & 0 \\
\hline
\end{tabular}

Poisson cluster, and antihyperuniform hyperplane intersection processes. Among the hyperuniform point processes, we characterize hypercubic lattices, randomly perturbed lattices, and stealthy disordered hyperuniform systems. We show that our simulation results for $\gamma_{1}(R)$, $\gamma_{2}(R)$, and $P[N(R)]$ for all models are in excellent agreement with the aforementioned rigorous bounds and exact results for the applicable ranges of $R$. For all disordered hyperuniform models, our explicit general formulas of these quantities in terms of $n$-body information enable us to infer the existence of "hidden" order that manifests itself, for the first time, at the three-body level or higher.

For each model considered in this paper, we are interested in ascertaining how large $R$ must be such that $P[N(R)]$ is well approximated by the Gaussian (normal) distribution. We have found that such "distance" metrics proposed previously are not adequate for assessing the Gaussianity of the diverse set of models that we consider here across dimensions. We quantify this proximity to the normal distribution for any model by introducing a certain Gaussian distance metric $l_{2}(R)$, defined in Sec. VII. This distance metric enables us to accurately determine when the distribution function $P[N(R)]$ for a particular model is tending to a CLT [71]. Because the distributions for all models (except the lattices) across dimensions are unimodal, the tendency to a CLT corresponds to the skewness and excess kurtosis simultaneously tending to zero. We have found that almost all of the considered models across dimensions obey a CLT. The convergence to a CLT is slowest for the antihyperuniform point process, followed by the Poisson cluster process and the Poisson process. The nonhyperuniform RSA and equilibrium packings tend to a CLT at the same rate as a Poisson point process but with smaller coefficients of proportionality. Among all models, the convergence to a CLT is generally fastest for the disordered hyperuniform processes. The only models considered that do not achieve a CLT are the hypercubic lattices for any $d$ and 1D hyperuniform systems of class I. The reader is referred to Sec. VII for details.

We have examined a variety of well-known closed-form probability distributions $P[N(R)]$ to ascertain those which best approximate the actual distributions for finite $R$ for all 
TABLE II. Other physical systems that are expected to display the salient fluctuation phenomena exhibited by the various models considered in this work.

\begin{tabular}{|c|c|c|}
\hline Models & & Physical systems \\
\hline Antihyperuniform & HIP & $\begin{array}{l}\text { Systems at thermal critical points [41-46]; telecommunication networks in urban } \\
\text { environments [26]. }\end{array}$ \\
\hline \multirow[t]{4}{*}{$\begin{array}{l}\text { Standard } \\
\text { Nonhyperuniform }\end{array}$} & Poisson cluster & $\begin{array}{l}\text { Materials science (recrystallization, residual stresses) [47], galaxy distributions [48], and } \\
\text { ecology; see Refs. [49,50] and references therein. }\end{array}$ \\
\hline & Poisson & $\begin{array}{l}\text { Dilute gases, detection of alpha particles, and trees in forests; see Refs. [24,49,51,52] and } \\
\text { references therein. }\end{array}$ \\
\hline & RSA & $\begin{array}{l}\text { Nonequilibrium systems, such as cement paste [53]; ion implantation in semiconductors [54]; } \\
\text { protein adsorption [55]; polymer oxidation [56]; and particles in cell membranes [57]. }\end{array}$ \\
\hline & $\begin{array}{l}\text { Equil. hard } \\
\text { spheres }\end{array}$ & $\begin{array}{l}\text { Dense granular systems; simple and molecular liquids with strong repulsive interactions (e.g., } \\
\text { Lennard Jones models [52] or water [58,59]); and colloids [60]; see also Refs. [29,52,58] } \\
\text { and references therein. }\end{array}$ \\
\hline \multirow[t]{3}{*}{ Hyperuniform } & Defective crystals & Low-temperature imperfections in crystals (e.g., vacancies and phonons) [61-66]. \\
\hline & Stealthy & $\begin{array}{l}\text { Antenna array designs [67], random quantum cascade laser [68], and disordered photonic } \\
\text { networks [69]; see Ref. [16] for more examples. }\end{array}$ \\
\hline & Crystals & All perfect crystals $[64,65,70]$. \\
\hline
\end{tabular}

of our models. Interestingly, we discover a type of universality in that, for all of our models that obey a CLT, the gamma distribution provides a good approximation to the number distribution $P[N(R)]$ across all dimensions for intermediate to large values of $R$ (Sec. VII C). It is noteworthy that, since the gamma distribution is determined by two parameters, this approximate universality makes it possible to estimate, based only on the first two moments, the large- $R$ scalings of $\gamma_{1}(R), \gamma_{2}(R)$, and $l_{2}(R)$ for all models across dimensions that obey a CLT. Among all models, the convergence to a CLT is generally fastest for the disordered hyperuniform processes such that $\gamma_{1}(R) \sim l_{2}(R) \sim R^{-(d+1) / 2}$ and $\gamma_{2}(R) \sim R^{-(d+1)}$ for large $R$. For standard nonhyperuniform models, convergence to a CLT is slower, such that $\gamma_{1}(R) \sim l_{2}(R) \sim R^{-d / 2}$ and $\gamma_{2}(R) \sim R^{-d}$. Finally, convergence to a CLT is slowest for the antihyperuniform model such that $\gamma_{1}(R) \sim l_{2}(R) \sim$ $R^{-1 / 2}$ and $\gamma_{2}(R) \sim R^{-1}$. These predictions are corroborated by the corresponding simulation results.

To answer the question what is the effect of increasing the space dimension on the number fluctuations for any particular model, we recall the so-called decorrelation principle [72], which roughly states that for many disordered point process, unconstrained correlations that exist in low dimensions vanish as $d$ tends to infinity, and all higher-order correlation functions $g_{n}$ for $n \geq 3$ may be expressed in terms of the number density $\rho$ and pair correlation function $g_{2}$. The decorrelation principle is employed to justify the conjecture that the densest sphere packings in sufficiently high dimensions are disordered (as opposed to ordered in low dimensions) [72,73]. Importantly, decorrelation in pair statistics has been shown to manifest itself in low dimensions in the case of disordered sphere packings [38,74,75] as well as other disordered systems with strongly repulsively interacting particles [76,77]. Since the number distribution function $P[N(R)]$ generally involves certain integrals over all of the $n$-body correlation functions, the decorrelation principle implies that $P[N(R)]$ increasingly becomes Gaussian-like as the space dimension increases for any model that decorrelates with $d$. Similarly, for models that correlate with $d, P[N(R)]$ increasingly deviates from the normal distribution as $d$ increases, as verified by computing the pair correlation function given by Eq. (79). We confirm such behaviors for all models that obey a CLT (Sec. VII D).

In Sec. II, we provide basic definitions and necessary background material. In Sec. III, we derive explicit closedform integral expressions for $\gamma_{1}(R)$ and $\gamma_{2}(R)$ as well as rigorous lower bounds on both of these quantities. We also obtain some general exact results for the first few cumulants and distribution functions for sphere packings, whether disordered or not. Section IV describes the large variety of nonhyperuniform and hyperuniform models in one, two, and three dimensions that we study in this paper. In Sec. V, we discuss our proposed Gaussian distance metric. Section VI describes the simulation procedure that we employ to sample the first four cumulants and number distributions. In Sec. VII, we present our results. We make concluding remarks in Sec. VIII.

\section{DEFINITIONS AND BACKGROUND}

A stochastic point process in $\mathbb{R}^{d}$ is defined as a mapping from a probability space to configurations of points $\mathbf{r}_{1}, \mathbf{r}_{2}, \mathbf{r}_{3} \ldots$ in $d$-dimensional Euclidean space $\mathbb{R}^{d}$; see Ref. [49] for mathematical details. Let $X$ denote the 
set of configurations such that each configuration $x \in X$ is a subset of $\mathbb{R}^{d}$ that satisfies two regularity conditions: (i) There are no multiple points, i.e., no points coincide $\left(\mathbf{r}_{i} \neq \mathbf{r}_{j}\right.$ if $i \neq j$ ), and (ii) each bounded subset of $\mathbb{R}^{d}$ must contain only a finite number of points of $x$ (i.e., $x$ is "locally finite"). The point process is statistically characterized by the generic $n$-particle probability density function $\rho_{n}\left(\mathbf{r}^{n}\right)$, where $\mathbf{r}^{n}$ is a shorthand notation for the position vectors of any $n$ points, i.e., $\mathbf{r}^{n} \equiv \mathbf{r}_{1}, \mathbf{r}_{2}, \ldots, \mathbf{r}_{n}[5,29]$. In other words, the quantity $\rho_{n}\left(\mathbf{r}^{n}\right) d \mathbf{r}^{n}$ is proportional to the probability of finding any $n$ particles with configuration $\mathbf{r}^{\mathbf{n}}$ in volume element $d \mathbf{r}^{n} \equiv d \mathbf{r}_{1} d \mathbf{r}_{2} \cdots d \mathbf{r}_{n}$; i.e., it is the probability measure. For any subvolume $\Omega \in \mathbb{R}^{d}$, the following normalization (average) condition involving the fluctuating number of particles within this subvolume, $N_{\Omega}$, immediately follows:

$$
\left\langle\frac{N_{\Omega} !}{\left(N_{\Omega}-n\right) !}\right\rangle_{\Omega}=\int_{\Omega} \int_{\Omega} \cdots \int_{\Omega} \rho_{n}\left(\mathbf{r}^{n}\right) d \mathbf{r}^{n} .
$$

Note that this random setting is quite general; it incorporates cases in which the locations of the points are deterministically known, such as a lattice.

For statistically homogeneous media, $\rho_{n}\left(\mathbf{r}^{n}\right)$ is translationally invariant and hence depends only on the relative displacements, say, with respect to $\mathbf{r}_{1}$ :

$$
\rho_{n}\left(\mathbf{r}^{n}\right)=\rho_{n}\left(\mathbf{r}_{12}, \mathbf{r}_{13}, \ldots, \mathbf{r}_{1 n}\right),
$$

where $\mathbf{r}_{i j}=\mathbf{r}_{j}-\mathbf{r}_{i}$. In particular, the one-body function $\rho_{1}$ is just equal to the constant number density of particles $\rho$. For statistically homogeneous point patterns, it is convenient to define the so-called $n$-body correlation function

$$
g_{n}\left(\mathbf{r}^{n}\right)=\frac{\rho_{n}\left(\mathbf{r}^{n}\right)}{\rho^{n}} .
$$

In systems without long-range order and in which the particles are mutually far from one another, $\rho_{n}\left(\mathbf{r}^{n}\right) \rightarrow \rho^{n}$, and we have from Eq. (9) that $g_{n}\left(\mathbf{r}^{n}\right) \rightarrow 1$. Thus, the deviation of $g_{n}$ from unity provides a measure of the degree of spatial correlation between the particles, with unity corresponding to no spatial correlation.

The important two-body quantity $g_{2}\left(\mathbf{r}_{12}\right)$ is usually referred to as the pair correlation function. The total correlation function $h\left(\mathbf{r}_{12}\right)$ is defined as

$$
h\left(\mathbf{r}_{12}\right)=g_{2}\left(\mathbf{r}_{12}\right)-1,
$$

and thus is a function that is zero when there are no spatial correlations in the system. Observe that the structure factor $S(\mathbf{k})$ is related to the Fourier transform of $h(\mathbf{r})$, denoted by $\tilde{h}(\mathbf{k})$, via the expression

$$
S(\mathbf{k})=1+\rho \tilde{h}(\mathbf{k})
$$

where

$$
\tilde{h}(\mathbf{k})=\int_{\mathbb{R}^{d}} h(\mathbf{r}) e^{-i \mathbf{k} \cdot \mathbf{r}} d \mathbf{r} .
$$

A hyperuniform point process is one in which singlescattering events at infinite wavelength vanish, i.e.,

$$
\lim _{|\mathbf{k}| \rightarrow 0} S(\mathbf{k})=0 .
$$

This property implies that a hyperuniform system obeys the following sum rule in direct space:

$$
\rho \int_{\mathbb{R}^{d}} h(\mathbf{r}) d \mathbf{r}=-1,
$$

and hence $h(\mathbf{r})$ must exhibit negative pair correlations, i.e., anticorrelations, for some values of $\mathbf{r}$ [16]. By contrast, an antihyperuniform point process is one in which $S(\mathbf{k})$ tends to $+\infty$ in the limit $|\mathbf{k}| \rightarrow 0$ [16].

A lattice $\Lambda$ in $\mathbb{R}^{d}$ is a subgroup consisting of the integer linear combinations of independent vectors that span $\mathbb{R}^{d}$ and thus, it represents a special subset of point processes. In a lattice $\Lambda$, the space $\mathbb{R}^{d}$ can be geometrically divided into identical regions $F$ called fundamental cells, each of which contains just one point specified by the lattice vector $[70,78]$. In the physical sciences, a lattice is equivalent to a Bravais lattice. Unless otherwise stated, we use the term lattice. A periodic point process is a more general notion than a lattice because it is obtained by placing a fixed configuration of $N$ points (where $N \geq 1$ ), called the basis, within one fundamental cell of a lattice $\Lambda$, which is then periodically replicated. Thus, the point process is still periodic under translations by $\Lambda$, but the $N$ points can occur anywhere in the chosen fundamental cell. Any lattice or periodic point configuration can be made statistically homogeneous by uniform translations of the pattern within the fundamental cell.

We call a packing in $\mathbb{R}^{d}$ a collection of nonoverlapping particles [79]. The centroids of the particles constitute a special point process in which no two particles can be closer than some minimal distance. In this paper, we consider packings of identical spheres of diameter $D$. The packing fraction $\phi=\rho v_{1}(D / 2)$ is the fraction of space covered by the spheres, where $v_{1}(R)$ is the volume of a sphere of radius $R$ given by Eq. (1). Any periodic point configuration with a finite basis can be regarded as a packing since there is a minimal pair distance.

\section{GENERAL LOCAL MOMENT FORMULAS AND PROBABILITY DISTRIBUTION FOR HOMOGENEOUS POINT PROCESSES}

We consider the determination of local moment formulas using the formalism of Torquato and Stillinger [13] that was used to obtain formulas for the local number variance. Here, we immediately begin with a $d$-dimensional spherical 
window of radius $R$ in $d$-dimensional Euclidean space $\mathbb{R}^{d}$, with the window indicator function

$$
w\left(\mathbf{r}-\mathbf{x}_{0} ; R\right)= \begin{cases}1 & \left|\mathbf{r}-\mathbf{x}_{0}\right| \leq R \\ 0 & \left|\mathbf{r}-\mathbf{x}_{0}\right|>R\end{cases}
$$

where $\mathbf{r}$ is some arbitrary position vector in $\mathbb{R}^{d}$ and $\mathbf{x}_{0}$ is the position vector of the window center. The number of points $N\left(R ; \mathbf{x}_{0}\right)$ within the window centered at position $\mathbf{x}_{0}$ is given by

$$
N\left(R ; \mathbf{x}_{0}\right)=\sum_{i=1} w\left(\mathbf{r}_{i}-\mathbf{x}_{0} ; R\right)
$$

which must be a finite number. We subsequently use the fact that

$$
v_{n}^{\mathrm{int}}\left(\mathbf{r}^{n} ; R\right)=\int_{\mathbb{R}^{d}} \prod_{i=1}^{n} w\left(\mathbf{r}_{i}-\mathbf{x}_{0} ; R\right) d \mathbf{x}_{0}
$$

is the intersection volume of $n$ spheres of radius $R$ centered at positions $\mathbf{r}_{1}, \mathbf{r}_{2}, \ldots, \mathbf{r}_{n}$.

\section{A. Moments}

The $m$ th moment associated with the random variable $N(R)$ is given by the following ensemble average:

$$
\begin{aligned}
\left\langle N^{m}(R)\right\rangle= & \left\langle\sum_{i_{1}=1} \sum_{i_{2}=1} \cdots \sum_{i_{m}=1} w\left(\mathbf{r}_{i_{1}}-\mathbf{x}_{0} ; R\right) w\left(\mathbf{r}_{i_{2}}-\mathbf{x}_{0} ; R\right)\right. \\
& \left.\cdots \times w\left(\mathbf{r}_{i_{m}}-\mathbf{x}_{0} ; R\right)\right\rangle .
\end{aligned}
$$

Here, we have implicitly assumed homogeneity, which renders the $m$ th moment independent of the position of the window $\mathbf{x}_{0}$. Under the ergodic assumption, the ensemble average indicated on the left-hand side of relation (18) is equivalent to averaging by uniformly window sampling a single realization over the infinite space.

Following the same procedure used in Ref. [13] to obtain an explicit formula for the second moment, we obtain from Eq. (18) that the $m$ th moment $\left\langle N^{m}(R)\right\rangle$ for a homogeneous process is given by integrals involving the finite set of correlation functions $g_{2}, g_{3}, \ldots, g_{m}$ weighted with the set of intersection volumes $v_{2}^{\text {int }}, v_{3}^{\text {int }}, \ldots, v_{m}^{\text {int }}$. For example, expanding the sums in Eq. (18) into one-body, two-body, ... and $m$-body terms in a manner analogous to the one given in Ref. [13], third and fourth moments are explicitly given by

$$
\begin{aligned}
\left\langle N^{3}(R)\right\rangle= & \rho v_{1}(R)+3 \rho^{2} \int_{\mathbb{R}^{d}} g_{2}(\mathbf{r}) v_{2}^{\text {int }}(r ; R) d \mathbf{r} \\
& +\rho^{3} \int_{\mathbb{R}^{d}} \int_{\mathbb{R}^{d}} g_{3}\left(\mathbf{r}^{3}\right) v_{3}^{\text {int }}\left(\mathbf{r}^{3} ; R\right) d \mathbf{r}_{2} d \mathbf{r}_{3}
\end{aligned}
$$

and

$$
\begin{aligned}
\left\langle N^{4}(R)\right\rangle= & \rho v_{1}(R)+7 \rho^{2} \int_{\mathbb{R}^{d}} g_{2}(\mathbf{r}) v_{2}^{\text {int }}(r ; R) d \mathbf{r} \\
& +6 \rho^{3} \int_{\mathbb{R}^{d}} \int_{\mathbb{R}^{d}} g_{3}\left(\mathbf{r}^{3}\right) v_{3}^{\text {int }}\left(\mathbf{r}^{3} ; R\right) d \mathbf{r}_{2} d \mathbf{r}_{3} \\
& +\rho^{4} \int_{\mathbb{R}^{d}} \int_{\mathbb{R}^{d}} \int_{\mathbb{R}^{d}} g_{4}\left(\mathbf{r}^{4}\right) v_{4}^{\text {int }}\left(\mathbf{r}^{4} ; R\right) d \mathbf{r}_{2} d \mathbf{r}_{3} d \mathbf{r}_{4},
\end{aligned}
$$

where $v_{n}^{\text {int }}$ is given by Eq. (17).

We are generally interested in the $m$ th-order cumulant $C_{m}(R)$, which is directly related to the $m$ th central moment $\left\langle[N(R)-\langle N(R)\rangle]^{m}\right\rangle$. For example, the first several cumulants are given by

$$
\begin{aligned}
C_{2}(R) & \equiv \sigma^{2}(R) \\
& =\rho v_{1}(R)+\rho^{2} \int_{\mathbb{R}^{d}} h(\mathbf{r}) v_{2}^{\text {int }}(r ; R) d \mathbf{r} \\
& =\rho v_{1}(R)\left[1-\rho v_{1}(R)\right]+\rho^{2} \int_{\mathbb{R}^{d}} g_{2}(\mathbf{r}) v_{2}^{\text {int }}(r ; R) d \mathbf{r}, \\
C_{3}(R) & \equiv\left\langle[N(R)-\langle N(R)\rangle]^{3}\right\rangle \\
& =\left\langle N^{3}(R)\right\rangle-3\left\langle N^{2}(R)\right\rangle\langle N(R)\rangle+2\langle N(R)\rangle^{3} \\
& =\rho v_{1}(R)+3 \rho^{2} \int_{\mathbb{R}^{d}} h(\mathbf{r}) v_{2}^{\text {int }}(r ; R) d \mathbf{r} \\
& +\rho^{3} \int_{\mathbb{R}^{d}} \int_{\mathbb{R}^{d}}\left[g_{3}\left(\mathbf{r}^{3}\right)-3 g_{2}\left(\mathbf{r}_{12}\right)+2\right] v_{3}^{\text {int }}\left(\mathbf{r}^{3} ; R\right) d \mathbf{r}_{2} d \mathbf{r}_{3},
\end{aligned}
$$

and

$$
\begin{aligned}
C_{4}(R) \equiv & \left\langle[N(R)-\langle N(R)\rangle]^{4}\right\rangle-3 \sigma^{4}(R) \\
= & \left\langle N^{4}(R)\right\rangle-4\left\langle N^{3}(R)\right\rangle\langle N(R)\rangle-3\left\langle N^{2}(R)\right\rangle^{2}+12\left\langle N^{2}(R)\right\rangle\langle N(R)\rangle^{2}-6\langle N(R)\rangle^{4} \\
= & \rho v_{1}(R)+7 \rho^{2} \int_{\mathbb{R}^{d}} h(\mathbf{r}) v_{2}^{\text {int }}(r ; R) d \mathbf{r}+6 \rho^{3} \int_{\mathbb{R}^{d}} \int_{\mathbb{R}^{d}}\left[g_{3}\left(\mathbf{r}^{3}\right)-3 g_{2}\left(\mathbf{r}_{12}\right)+2\right] v_{3}^{\operatorname{int}}\left(\mathbf{r}^{3} ; R\right) d \mathbf{r}_{2} d \mathbf{r}_{3} \\
& +\rho^{4} \int_{\mathbb{R}^{d}} \int_{\mathbb{R}^{d}} \int_{\mathbb{R}^{d}}\left[g_{4}\left(\mathbf{r}^{4}\right)-4 g_{3}\left(\mathbf{r}^{3}\right)+12 g_{2}\left(\mathbf{r}_{12}\right)-6\right] v_{4}^{\text {int }}\left(\mathbf{r}^{4} ; R\right) d \mathbf{r}_{2} d \mathbf{r}_{3} d \mathbf{r}_{4}-3\left[\rho^{2} \int_{\mathbb{R}^{d}} g_{2}(\mathbf{r}) v_{2}^{\text {int }}(r ; R) d \mathbf{r}\right]^{2},
\end{aligned}
$$


where we have used the following identities:

$$
\begin{gathered}
v_{1}(R) v_{2}^{\text {int }}\left(r_{12} ; R\right)=\int_{\mathbb{R}^{d}} v_{3}^{\text {int }}\left(\mathbf{r}^{3} ; R\right) d \mathbf{r}_{3}, \\
v_{1}^{3}(R)=\int_{\mathbb{R}^{d}} \int_{\mathbb{R}^{d}} v_{3}^{\text {int }}\left(\mathbf{r}^{3} ; R\right) d \mathbf{r}_{2} d \mathbf{r}_{3}, \\
v_{1}(R) v_{3}^{\text {int }}\left(\mathbf{r}^{3} ; R\right)=\int_{\mathbb{R}^{d}} v_{4}^{\text {int }}\left(\mathbf{r}^{4} ; R\right) d \mathbf{r}_{4}, \\
v_{1}^{2}(R) v_{2}^{\text {int }}\left(r_{12} ; R\right)=\int_{\mathbb{R}^{d}} \int_{\mathbb{R}^{d}} v_{4}^{\text {int }}\left(\mathbf{r}^{4} ; R\right) d \mathbf{r}_{3} d \mathbf{r}_{4}, \\
v_{1}^{4}(R)=\int_{\mathbb{R}^{d}} \int_{\mathbb{R}^{d}} \int_{\mathbb{R}^{d}} v_{4}^{\text {int }}\left(\mathbf{r}^{4} ; R\right) d \mathbf{r}_{2} d \mathbf{r}_{3} d \mathbf{r}_{4} .
\end{gathered}
$$

We now derive a lower bound on $C_{3}(R)$ in terms of the first and second cumulants for general point processes. This bound easily follows from the fact that the product $g_{3}\left(\mathbf{r}^{3}\right) v_{3}^{\text {int }}\left(\mathbf{r}^{3} ; R\right)$ in the last integral of Eq. (22) is nonnegative for all positions, so dropping this integral yields the following lower bound:

$$
\begin{aligned}
C_{3}(R) \geq & \rho v_{1}(R)\left[1-\rho v_{1}(R)\right]\left[1-2 \rho v_{1}(R)\right] \\
& +3\left[1-\rho v_{1}(R)\right]\left(\sigma^{2}(R)-\rho v_{1}\left[1-\rho v_{1}(R)\right]\right) .
\end{aligned}
$$

Similarly, dropping the positive integral in Eq. (23) involving the product $g_{4}\left(\mathbf{r}^{4}\right) v_{4}^{\text {int }}\left(\mathbf{r}^{4} ; R\right)$ gives the following lower bound on $C_{4}(R)$ in terms of the first, second, and third cumulants:

$$
\begin{aligned}
C_{4}(R) \geq & \rho v_{1}(R)\left[1-\rho v_{1}(R)\right]\left[2-\rho v_{1}(R)\right]\left[3-\rho v_{1}(R)\right] \\
& -\sigma^{2}(R)\left(11-18 \rho v_{1}(R)+6\left[\rho v_{1}(R)\right]^{2}\right) \\
& -3 \sigma^{4}+2 C_{3}(R)\left[3-2 \rho v_{1}(R)\right] .
\end{aligned}
$$

These bounds are relatively tight for sufficiently small values of $R$ and can be exact (sharp) for such $R$ for packings, as discussed in Sec. III C.

The third- and fourth-order cumulants are directly related to the skewness and excess kurtosis, respectively. The skewness is often defined as

$$
\gamma_{1}(R) \equiv \frac{C_{3}(R)}{\sigma^{3}(R)}
$$

which, qualitatively speaking, is a measure of the asymmetry of the probability distribution. Positive or negative skewness means that the distribution has a heavier right or left tail, respectively. The excess kurtosis, which is a measure of the heaviness of the "tails" of the probability distribution, is defined as

$$
\gamma_{2}(R) \equiv \frac{C_{4}(R)}{\sigma^{4}(R)}
$$

Positive or negative excess kurtosis means heavier or lighter tails than Gaussian, respectively. Both skewness and excess kurtosis are identically zero for the normal distribution.

For a general random variable, Pearson derived a lower bound on the excess kurtosis in terms of the skewness [80]:

$$
\gamma_{2}(R) \geq \gamma_{1}^{2}(R)-2 .
$$

We also apply this lower bound to validate our numerical results for all of our model point processes. Such details are reported in the Supplemental Material (SM) [81].

Both $\gamma_{1}$ and $\gamma_{2}$ are identically zero for the normal distribution, and hence, such lower-order information can herald at what value of $R$ a general point configuration can be approximated by a normal distribution. Note that the higher-order cumulants become increasingly more complicated but can be written as a determinant involving the moments $\left\langle N^{m}(R)\right\rangle$ [82].

In the case of a homogeneous Poisson point process, $g_{n}=1$ for all $n$. Hence, from the expressions above, it immediately follows that $C_{2}(R)=C_{3}(R)=C_{4}(R)=\rho v_{1}(R)$, which are the expected well-known results for this point process. It follows that the corresponding skewness and excess kurtosis are exactly given by

$$
\gamma_{1}(R)=\frac{1}{\left[\rho v_{1}(R)\right]^{1 / 2}} \propto R^{-d / 2}
$$

and

$$
\gamma_{2}(R)=\frac{1}{\rho v_{1}(R)} \propto R^{-d},
$$

respectively. Observe that both $\gamma_{1}(R)$ and $\gamma_{2}(R)$ tend to zero in the limit $R \rightarrow \infty$, which is consistent with the fact that the Poisson point process obeys a CLT.

\section{B. Probability distribution function}

It is straightforward to show that for an arbitrarily shaped window region $\Omega$, the probability distribution function is given by $[2,3]$

$$
P\left[N_{\Omega}=M\right]=\sum_{m=M}^{\infty}(-1)^{m-M} \frac{\mathcal{A}_{m}(\Omega)}{M !(m-M) !},
$$

where

$$
\begin{aligned}
\mathcal{A}_{m}(\Omega) & \equiv\left\langle\frac{N_{\Omega} !}{\left(N_{\Omega}-m\right) !}\right\rangle_{\Omega} \\
& =\rho^{m} \int_{\Omega} \int_{\Omega} \ldots \int_{\Omega} g_{m}\left(\mathbf{r}^{m}\right) d \mathbf{r}_{1} d \mathbf{r}_{2} \ldots d \mathbf{r}_{m}
\end{aligned}
$$

is exactly the same as the average given in Eq. (7) under the assumption of homogeneity. Thus, we see that the average 
$\mathcal{A}_{m}(\Omega)$ is the nontrivial and common contribution to $P\left[N_{\Omega}\right]$ for any specific value of $N_{\Omega}$. The only differences in $P\left[N_{\Omega}\right]$ for different values of $N_{\Omega}$ are the combinatoric factors multiplying the coefficients $\mathcal{A}_{m}(\Omega)$ in the series (36). Because these coefficients are intrinsically positive, relation (36) is an alternating series.

When $\Omega$ is a spherical window of radius $R$, it simply follows that

$$
P[N(R)=M]=\sum_{m=M}^{\infty}(-1)^{m-M} \frac{\mathcal{A}_{m}(R)}{M !(m-M) !},
$$

where

$$
\begin{aligned}
\mathcal{A}_{m}(R) \equiv & \left\langle\frac{N(R) !}{(N(R)-m) !}\right\rangle_{v_{1}(R)} \\
= & \rho^{m} \int_{\mathbb{R}^{d}} \int_{\mathbb{R}^{d}} \ldots \int_{\mathbb{R}^{d}} g_{m}\left(\mathbf{r}^{m}\right) \\
& \times v_{m}^{\mathrm{int}}\left(\mathbf{r}^{m} ; R\right) d \mathbf{r}_{2} d \mathbf{r}_{3} \ldots d \mathbf{r}_{m} .
\end{aligned}
$$

For a fixed value of $M \in \mathbb{N}_{0}, P[N(R)<M]$ as a function of $R$ is a complementary cumulative distribution function, which is associated with the "void" probability density function $H_{V}(R ; M)$ [9], where $H_{V}(R ; M) d R$ is the probability that the distance to the $M$ th nearest neighbor from an arbitrary position in space is between $R$ and $R+d R$. For example, in the case $M=1, P[N(R)<1]=P[N(R)=0]$ is the well-known void exclusion probability function $E_{V}(R)$, which is associated with the void nearest-neighbor probability density function $H_{V}(R)[29,78,83]$. In the case of a homogeneous Poisson point process, we can immediately recover the exact result (6) for the number distribution from relations (38) and (39) using the fact that $g_{n}=1$ for all $n$ and the identity

$v_{1}(R)^{m}=\int_{\mathbb{R}^{d}} \int_{\mathbb{R}^{d}} \ldots \int_{\mathbb{R}^{d}} v_{m}^{\text {int }}\left(\mathbf{r}^{m} ; R\right) d \mathbf{r}_{2} d \mathbf{r}_{3} \ldots d \mathbf{r}_{m}$.

Exact results for $P[N(R)]$ for non-Poissonian point processes are rare. One exception is the one-dimensional model of equilibrium hard rods for which $P[N(R)]$ is known exactly [9]. In the case of disordered equilibrium hard-disk $(d=2)$ and hard-sphere $(d=3)$ packings, accurate but approximate expressions for the exclusion probability $E_{V}(R) \equiv P[N(R)=0]$ are available $[29,83]$. Thus, in principle, one can extract from such formulas approximations for $\mathcal{A}_{m}(R)$ to get the corresponding approximations for $P[N(R)]$ for any $N(R) \geq 1$ for such packings. The difficulty in ascertaining $P[N(R)]$ exactly for nontrivial models can be appreciated by appealing to the ghost random sequential addition (RSA) packing process [72], for which the $g_{m}$ are known exactly for any $m$. The ghost RSA process modifies the standard time-dependent RSA algorithm in the following way: A test sphere at time $t$ is accepted only if it does not overlap an existing sphere in the packing or any previously rejected test spheres (which are called "ghost" spheres). While the evaluation of the integral (39) for ghost RSA can be carried out exactly for very small $m$, closed form expressions are not available for general values of $m$, which points to the importance of devising accurate numerical methods to determine the number distributions of packings. For example, $P[N(R)]$ has be determined in simulations for equilibrium hard spheres and maximally random jammed (MRJ) sphere packings in Ref. [15].

It has already been established rigorously that truncations of the alternating series (38) for the special case $P[N(R)=0]=E_{V}(R)$ at an even and odd number of terms yield successive upper and lower bounds on the probability distribution $P[N(R)=0]$, respectively. Such bounds are consequences of an inclusion-exclusion principle associated with this alternating series. Here, we make the simple observation that the same inclusion-exclusion principle applies for any value of $N(R)$. The first several of such bounds are given by

$$
\begin{gathered}
P[N(R)] \leq \frac{\mathcal{A}_{N}(R)}{N(R) !} \\
P[N(R)] \geq \frac{\mathcal{A}_{N}(R)}{N(R) !}-\frac{\mathcal{A}_{N+1}(R)}{N(R) !} \\
P[N(R)] \leq \frac{\mathcal{A}_{N}(R)}{N(R) !}-\frac{\mathcal{A}_{N+1}(R)}{N(R) !}+\frac{\mathcal{A}_{N+2}(R)}{2 ! N(R) !}, \\
P[N(R)] \geq \frac{\mathcal{A}_{N}(R)}{N(R) !}-\frac{\mathcal{A}_{N+1}(R)}{N(R) !}+\frac{\mathcal{A}_{N+2}(R)}{2 ! N(R) !}-\frac{\mathcal{A}_{N+3}(R)}{3 ! N(R) !}
\end{gathered}
$$

where $\mathcal{A}_{N}(R)$ is shorthand for $\mathcal{A}_{N(R)}(R)$. These bounds become increasingly sharper as more terms are included. Moreover, these bounds can be sharp (exact) for sufficiently small $R$ for sphere packings, as discussed in Sec. III C. We utilize these bounds to validate our simulation results for all models across dimensions in the SM [81].

\section{Elementary results for packings}

Here, we obtain some general exact results for the first few cumulants and distribution functions for packings of identical spheres of diameter $D$, whether disordered or not. Results that apply to lattice packings are also derived.

Because no two spheres can overlap when their centers are separated by a distance less than or equal to $D$, the cumulants can be written explicitly for $d \geq 1$ and $R \leq D / 2$ since such a window region can accommodate, at most, a single sphere. For example, the integral involving $g_{2}$ in the last line of Eq. (21) is identically zero, and hence for $R \leq D / 2$, 


$$
C_{2}(R)=\rho v_{1}(R)\left[1-\rho v_{1}(R)\right],
$$

which was noted by Torquato and Stillinger [13]. More generally, noting that $\left\langle N^{m}(R)\right\rangle=\rho v_{1}(R)$ for all $m$ because all other terms in Eqs. (19) and (20) involve either $g_{2}, g_{3}$, or $g_{4}$, we have from Eqs. (22) and (23) for $d \geq 1$ that, for $R \leq D / 2$,

$$
\begin{gathered}
C_{3}(R)=\rho v_{1}(R)\left[1-\rho v_{1}(R)\right]\left[1-2 \rho v_{1}(R)\right], \quad(46) \\
C_{4}(R)=\rho v_{1}(R)\left[1-7 \rho v_{1}(R)+12 \rho^{2} v_{1}^{2}(R)-6 \rho^{3} v_{1}^{3}(R)\right] .
\end{gathered}
$$

Thus, for $R \leq D / 2$,

$$
\gamma_{1}(R)=\frac{1-2 \rho v_{1}(R)}{\left(\rho v_{1}(R)\left[1-\rho v_{1}(R)\right]\right)^{1 / 2}}
$$

and

$$
\gamma_{2}(R)=\frac{1-7 \rho v_{1}(R)+12 \rho^{2} v_{1}^{2}(R)-6 \rho^{3} v_{1}^{3}(R)}{\rho v_{1}(R)\left[1-\rho v_{1}(R)\right]^{2}} .
$$

As in the Poisson case, both the skewness $\gamma_{1}(R)$ and excess kurtosis $\gamma_{2}(R)$ diverge to $+\infty$ in the limit $R \rightarrow 0$. For $0 \leq R \leq D / 2$, while $\gamma_{1}(R)$ is generally a monotonically decreasing function of $R$ that can be non-negative, $\gamma_{2}(R)$ is generally a nonmonotonic function, but it can also be nonnegative. The corresponding results for the distribution function $P[N(R)]$ for $R \leq D / 2$ follow immediately from Eqs. (36) and (39), namely,

$$
P[N(R)=0]=1-\rho v_{1}(R),
$$

$$
P[N(R)=1]=\rho v_{1}(R),
$$

$$
P[N(R) \geq 2]=0 .
$$

When $D / 2<R \leq D / \sqrt{3}$ and $d \geq 2$, the window can accommodate at most two hard spheres. Therefore, following the same reasoning as above for $R \leq D / 2$, we find from Eqs. (22) and (23) that for $d \geq 2$ and $0 \leq R \leq D / \sqrt{3}$,

$$
\begin{aligned}
C_{3}(R) & =\rho v_{1}(R)+3 \rho^{2} \int_{\mathbb{R}^{d}} h(\mathbf{r}) v_{2}^{\text {int }}(r ; R) d \mathbf{r}-3 \rho^{3} v_{1}(R) \int_{\mathbb{R}^{d}} g_{2}\left(\mathbf{r}_{12}\right) v_{2}^{\text {int }}(r ; R) d \mathbf{r}+2 \rho^{3} v_{1}^{3}(R) \\
& =\rho v_{1}(R)\left[1-\rho v_{1}(R)\right]\left[1-2 \rho v_{1}(R)\right]+3\left[1-\rho v_{1}(R)\right] \rho^{2} \int_{\mathbb{R}^{d}} g_{2}(\mathbf{r}) v_{2}^{\text {int }}(r ; R) d \mathbf{r} \\
& =\rho v_{1}(R)\left[1-\rho v_{1}(R)\right]\left[1-2 \rho v_{1}(R)\right]+3\left[1-\rho v_{1}(R)\right]\left[\sigma^{2}(R)-\rho v_{1}\left[1-\rho v_{1}(R)\right]\right]
\end{aligned}
$$

and

$$
\begin{aligned}
C_{4}(R)= & \rho v_{1}(R)+7 \rho^{2} \int_{\mathbb{R}^{d}} h(\mathbf{r}) v_{2}^{\text {int }}(r ; R) d \mathbf{r}+6 \rho^{3} \int_{\mathbb{R}^{d}} \int_{\mathbb{R}^{d}}\left[-3 g_{2}\left(\mathbf{r}_{12}\right)+2\right] v_{3}^{\text {int }}\left(\mathbf{r}^{3} ; R\right) d \mathbf{r}_{2} d \mathbf{r}_{3} \\
& +\rho^{4} \int_{\mathbb{R}^{d}} \int_{\mathbb{R}^{d}} \int_{\mathbb{R}^{d}}\left[12 g_{2}\left(\mathbf{r}_{12}\right)-6\right] v_{4}^{\text {int }}\left(\mathbf{r}^{4} ; R\right) d \mathbf{r}_{2} d \mathbf{r}_{3} d \mathbf{r}_{4}-3\left[\rho^{2} \int_{\mathbb{R}^{d}} g_{2}(\mathbf{r}) v_{2}^{\text {int }}(r ; R) d \mathbf{r}\right]^{2} \\
= & \rho v_{1}(R)\left[1-7 \rho v_{1}(R)+12 \rho^{2} v_{1}^{2}(R)-6 \rho^{3} v_{1}^{3}(R)\right]+7 \rho^{2} \int_{\mathbb{R}^{d}} g_{2}(\mathbf{r}) v_{2}^{\text {int }}(r ; R) d \mathbf{r}-18 \rho^{3} v_{1}(R) \int_{\mathbb{R}^{d}} g_{2}(\mathbf{r}) v_{2}^{\text {int }}(r ; R) d \mathbf{r} \\
& +12 \rho^{4} v_{1}^{2}(R) \int_{\mathbb{R}^{d}} g_{2}(\mathbf{r}) v_{2}^{\text {int }}(r ; R) d \mathbf{r}-3\left[\rho^{2} \int_{\mathbb{R}^{d}} g_{2}(\mathbf{r}) v_{2}^{\text {int }}(r ; R) d \mathbf{r}\right]^{2} \\
= & \rho v_{1}(R)\left[1-7 \rho v_{1}(R)+12 \rho^{2} v_{1}^{2}(R)-6 \rho^{3} v_{1}^{3}(R)\right]+\left[7-18 \rho v_{1}(R)+12 \rho^{2} v_{1}^{2}(R)\right] \rho^{2} \int_{\mathbb{R}^{d}} g_{2}(\mathbf{r}) v_{2}^{\text {int }}(r ; R) d \mathbf{r} \\
& -3\left[\rho^{2} \int_{\mathbb{R}^{d}} g_{2}(\mathbf{r}) v_{2}^{\text {int }}(r ; R) d \mathbf{r}\right]^{2} \\
= & \rho v_{1}(R)\left[1-7 \rho v_{1}(R)+12 \rho^{2} v_{1}^{2}(R)-6 \rho^{3} v_{1}^{3}(R)\right]+\left[7-18 \rho v_{1}(R)+12 \rho^{2} v_{1}^{2}(R)\right]\left(\sigma^{2}(R)-\rho v_{1}(R)\left[1-\rho v_{1}(R)\right]\right) \\
& -3\left(\sigma^{2}(R)-\rho v_{1}(R)\left[1-\rho v_{1}(R)\right]\right)^{2} .
\end{aligned}
$$

We see that both $C_{3}(R)$ and $C_{4}(R)$ are given purely in terms of the mean $\langle N(R)\rangle=\rho v_{1}(R)$ and number variance $\sigma^{2}(R)$ for such $R$. Observe also that formula (53) is identical the lower bound (29) for general point processes. We note that for $R \leq D / 2$, relations (46) and (47) are recovered, as expected. Finally, for $d=1$, these formulas actually apply for $R \leq D$. 
When the window can accommodate at most three spheres, the fourth cumulant $C_{4}(R)$ for $0 \leq R \leq R_{*}(d)$ is exactly given in terms of the first three cumulants, i.e.,

$$
\begin{aligned}
C_{4}(R)= & \rho v_{1}(R)\left[1-\rho v_{1}(R)\right]\left[2-\rho v_{1}(R)\right]\left[3-\rho v_{1}(R)\right] \\
& -\sigma^{2}(R)\left(11-18 \rho v_{1}(R)+6\left[\rho v_{1}(R)\right]^{2}\right) \\
& -3 \sigma^{4}+2 C_{3}(R)\left[3-2 \rho v_{1}(R)\right],
\end{aligned}
$$

where $R_{*}(d)>D / \sqrt{3}$ is a threshold that depends on the space dimension $d$. For example, $R_{*}(1)=3 D / 2, R_{*}(2)=$ $\sqrt{2} D / 2$, and $R_{*}(3)=\sqrt{3 / 8} D$. Note that formula (55) is identical to the lower bound (30) for general point processes.

When the spherical window can accommodate at most two spheres, implying that $R \leq D / \sqrt{3}$ for $d \geq 2$, threebody and higher-order terms in the series (39) for the probability distribution vanish identically, yielding the following exact result for $P[N(R)]$ :

$$
\begin{gathered}
P[N(R)=0]=\left[1-\frac{1}{2} \rho v_{1}(R)\right]\left[1-\rho v_{1}(R)\right]+\frac{\sigma^{2}(R)}{2}, \\
P[N(R)=1]=2 \rho v_{1}(R)-\left[\rho v_{1}(R)\right]^{2}-\sigma^{2}(R), \\
P[N(R)=2]=\frac{\sigma^{2}(R)}{2}-\frac{\rho}{2} v_{1}(R)\left[1-\rho v_{1}(R)\right], \\
P[N(R) \geq 3]=0 .
\end{gathered}
$$

We see that for such windows, the entire distribution function is completely determined by the first and second cumulants. A nontrivial upper bound on the variance $\sigma^{2}(R)$ of a packing follows immediately from Eq. (57) and the fact that $P[N(R)=1]$ must be non-negative, i.e.,

$$
\sigma^{2}(R) \leq \rho v_{1}(R)\left[2-\rho v_{1}(R)\right] .
$$

For the same reasons, relation (58) yields the following general lower bound on the variance:

$$
\sigma^{2}(R) \geq \rho v_{1}(R)\left[1-\rho v_{1}(R)\right]
$$

As before, these formulas actually apply for $R \leq D$ for $d=1$.

Similarly, when the spherical window can accommodate at most three spheres, implying that $0 \leq R \leq R_{*}(d)$, fourbody and higher-order terms in the series (39) for $P[N(R)]$ vanish identically, yielding the following exact result for $P[N(R)]$ :

$$
\begin{aligned}
P[N(R)=0]= & 1-\rho v_{1}(R) \\
& -\frac{\rho v_{1}(R)}{6}\left[1-\rho v_{1}(R)\right]\left[5-\rho v_{1}(R)\right] \\
& +\frac{1}{2}\left[2-\rho v_{1}(R)\right] \sigma^{2}(R)-\frac{C_{3}(R)}{6}, \\
P[N(R)=1]= & \frac{1}{2} \rho v_{1}(R)\left[2-\rho v_{1}(R)\right]\left[3-\rho v_{1}(R)\right] \\
& +\frac{1}{2}\left[-5+3 \rho v_{1}(R)\right] \sigma^{2}(R)+\frac{1}{2} C_{3}(R),
\end{aligned}
$$

$$
\begin{aligned}
P[N(R)=2]= & -\frac{\rho v_{1}(R)}{2}\left[1-\rho v_{1}(R)\right]\left[3-\rho v_{1}(R)\right] \\
& +\frac{1}{2}\left[4-3 \rho v_{1}(R)\right] \sigma^{2}(R)-\frac{1}{2} C_{3}(R),
\end{aligned}
$$

$$
\begin{aligned}
P[N(R)=3]= & \frac{\rho v_{1}(R)}{6}\left[1-\rho v_{1}(R)\right]\left[2-\rho v_{1}(R)\right] \\
& -\frac{\sigma^{2}(R)}{2}\left[1-\rho v_{1}(R)\right]+\frac{C_{3}(R)}{6},
\end{aligned}
$$

$P[N(R) \geq 4]=0$.

Thus, for such $R$, the entire probability distribution function is completely determined by the first three cumulants. The non-negativities of the probabilities $P[N(R)=0]$ [Eq. (62)] and $P\left[N(R)=2\right.$ ] [Eq. (64)] yield upper bounds on $C_{3}(R)$ in terms of the first two cumulants, so the minimum of these two upper bounds is to be chosen. Similarly, the $P[N(R)=1]$ [Eq. (63)] and $P[N(R)=3$ ] [Eq. (65)] yield lower bounds on $C_{3}(R)$ in terms of the first two cumulants, so the maximum of these two lower bounds is to be chosen. In the SM [81], we demonstrate that the aforementioned exact results for $C_{3}(R), C_{4}(R)$, and certain $P[N(R)]$ are in excellent agreement with our corresponding simulation data for sphere packings examined in the paper across dimensions.

More generally, for any packing of identical spheres, a spherical region of radius $R$ can accommodate a maximum number of spheres, denoted by $N_{\max }(R)$. This maximal number can be determined from tabulations of the so-called densest local packings for a finite range of particle numbers in both two [84] and three [85] dimensions. Therefore, the number distribution $P[N(R)]$ for a packing is generally far from a normal distribution for a finite-sized window since it must have compact support such that it is zero for $N(R)>N_{\max }(R)$, i.e.,

$$
P[N(R)]=0 \quad \text { for } N(R)>N_{\max }(R) .
$$

In such instances, the distribution function is determined by a finite set of moments, i.e., the first, second, ..., $N_{\max }$ th moments. Moreover, for any dense packing or point 
process in which the nearest-neighbor distance is narrowly distributed (e.g., "strongly" stealthy systems described below and in Sec. IV B 3), $P[N(R)]$ will be zero for $N(R)<N_{\min }(R)$, where the cutoff value $N_{\min }(R)$ grows with $R$. This situation prevents a strict CLT from applying for finite-sized windows.

Another important observation is that for point processes in which the "hole" radius $R$ is bounded from above by $R_{\max }$, the probability of finding a spherical window with radius $R>R_{\max }$ must be zero, i.e., $P[N(R)=0]=0$ for $R>R_{\max }$, which of course is non-Gaussian behavior. The cutoff value $R_{\max }$ for a point process in $\mathbb{R}^{d}$ is its covering radius [78]. Processes with this bounded-hole property include periodic packings with a finite basis [86], quasicrystals [87], and the saturated random sequential addition packing process (see Sec. IVA 3). Disordered stealthy point processes also have bounded holes $[86,88]$, as discussed in Sec. IV B 3.

We note that for the hypercubic lattice $\mathbb{Z}^{d}$ scaled by $D$ (see Sec. IV B 1 for the precise definition), all of the relations derived above for the skewness, excess kurtosis, and distribution function for the situation $R \leq D / \sqrt{3}$ actually apply as well for the larger range $R \leq D / \sqrt{2}$ when $d \geq 2$, where $D$ is the lattice spacing. In fact, in the case of the scaled integer lattice $\rho^{-1} \mathbb{Z}$ at number density $\rho$, we can obtain an exact formula for $P[N(R)]$ by invoking the key idea of Ref. [13] to yield the exact result for the local number variance; namely, the number of points inside a window of radius $R$ can only take two values, either $N_{R}$ or $N_{R}+1$, where $N_{R} \equiv\lfloor 2 \rho R\rfloor$ and $\lfloor x\rfloor$ is the floor function of a real number $x$. The probability distribution for all $N(R)$ is given by

$$
\begin{gathered}
P\left[N(R)<N_{R}\right]=0, \\
P\left[N(R)=N_{R}\right]=1-\{2 \rho R\}, \\
P\left[N(R)=N_{R}+1\right]=\{2 \rho R\}, \\
P\left[N(R)>N_{R}+1\right]=0,
\end{gathered}
$$

where $\{x\} \equiv x-\lfloor x\rfloor$ is the fractional part of a positive number $x$. Thus, this skewed distribution is highly nonGaussian, with nonexistent left or right tails for almost all values of $N(R)$, implying values of the skewness and excess kurtosis that are generally far from zero for almost all values of $R$. From the distribution function (71) and relation (4), we can immediately obtain the first several cumulants:

$$
\begin{gathered}
\sigma^{2}(R)=\{2 \rho R\}(1-\{2 \rho R\}), \\
\gamma_{1}(R)=\frac{1-2\{2 \rho R\}}{[\{2 \rho R\}(1-\{2 \rho R\})]^{1 / 2}},
\end{gathered}
$$

$$
\gamma_{2}(R)=\frac{1-6\{2 \rho R\}(1-\{2 \rho R\})}{\{2 \rho R\}(1-\{2 \rho R\})}
$$

all of which are periodic functions of $2 \rho R$ with unit period. Relation (72) for the variance was given in Ref. [13]. The reader is referred to the top panel of Fig. 2, which shows plots of the variance, skewness, and excess kurtosis as a function of $R$ for $\mathbb{Z}$. The highly discrete nature of the number distribution for the integer lattice extends to that for the hypercubic lattice $\mathbb{Z}^{d}$ for $d \geq 2$, as we see in Sec. VII.

\section{NONHYPERUNIFORM AND HYPERUNIFORM MODELS}

We consider eight different models of statistically homogeneous point processes in two and three dimensions: five nonhyperuniform models, one of which is antihyperuniform (hyperplane intersection process or HIP), and three hyperuniform models. Analogous models are also examined in one dimension, except for the HIP, which is not defined in this dimension. Importantly, our models were chosen so that they span a representative subset of all possible ranges of $S(0)$. The reader is referred to Figs. 3 and 4 for representative images of configurations for each of the models in two dimensions. Table I lists the parameters of our models and simulations, including the values of $S(0)$, which is a crucially important way to classify them. We note that to compare all of our models in a consistent manner, we consider all of them to have unit number density $(\rho=1)$, which determines the units of distance [89].

It is useful to recall scaling relations for hyperuniform and nonhyperuniform point processes. Consider any homogeneous point process in $\mathbb{R}^{d}$ for which the structure factor has the following power-law behavior as the wave number tends to zero:

$$
S(\mathbf{k}) \sim|\mathbf{k}|^{\alpha} \quad(|\mathbf{k}| \rightarrow 0) .
$$

This scaling implies that the total correlation function $h(\mathbf{r})$ has the corresponding power-law behavior $1 /|\mathbf{r}|^{d+\alpha}$ for large $|\mathbf{r}|$ [16]. For hyperuniform systems, the exponent $\alpha$ is a positive constant, which implies that there are three different scaling regimes (classes) that describe the associated large- $R$ behaviors of the number variance $[13,16,90]$ :

$$
\sigma^{2}(R) \sim \begin{cases}R^{d-1} & \alpha>1 \quad(\text { Class I) } \\ R^{d-1} \ln R & \alpha=1 \quad(\text { Class II }) \\ R^{d-\alpha} & 0<\alpha<1 \quad(\text { Class III) }\end{cases}
$$

By contrast, for any nonhyperuniform system, it follows from the asymptotic analysis given in Ref. [16] that 

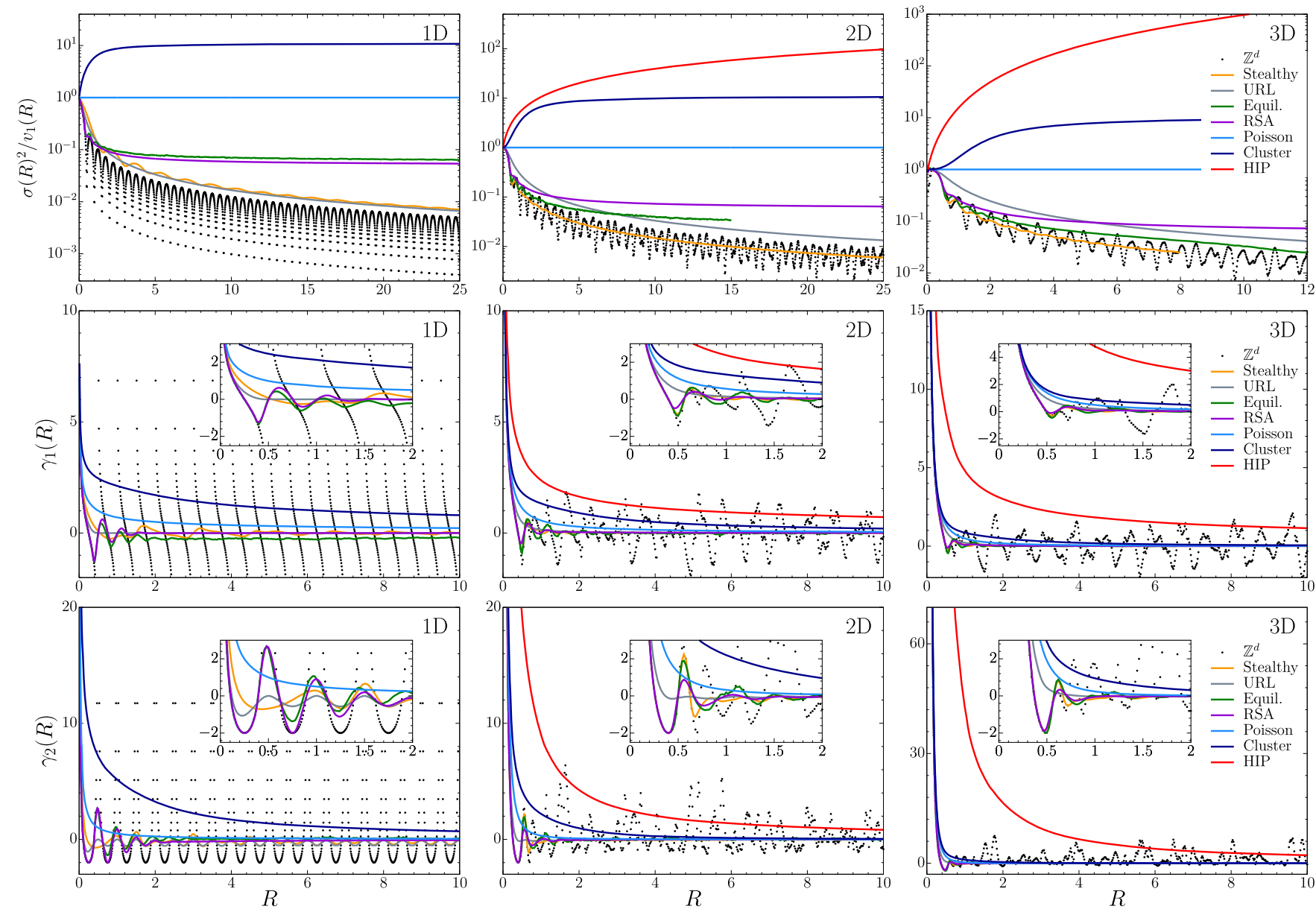

FIG. 2. Graphs of the number variance, skewness, and excess kurtosis versus the window radius $R$ for all considered models across the first three space dimensions.

$\sigma^{2}(R) \sim \begin{cases}R^{d} & \alpha=0 \quad \text { (typical nonhyperuniform) } \\ R^{d-\alpha} & -d<\alpha<0 \quad \text { (antihyperuniform) }\end{cases}$

The scaling for the antihyperuniform instance can be obtained by using an asymptotic analysis of either the direct-space representation (2) or the Fourier-space representation (3) of the number variance, as derived in Ref. [91]. The typical nonhyperuniform scaling in Eq. (77) results from the fact that $S(0)$ is bounded and, indeed, the implied constant multiplying $R^{d}$ is proportional to $S(0)$.

Any nonhyperuniform point process for which $S(0)>1$ has a large- $R$ asymptotic number variance $\sigma^{2}(R)$ that is larger than that for a Poisson point process $[S(0)=1]$ with the same mean $\langle N(R)\rangle$. We call such a nonhyperuniform point process "super-Poissonian." Two examples of super-Poissonian point processes studied in this work are the Poisson cluster and HIP point processes described below.

\section{A. Nonhyperuniform processes}

\section{Poisson point process}

A homogeneous Poisson point process in $\mathbb{R}^{d}$ has a structure factor $S(k)=1$ for all $k$ and hence is nonhyperuniform. At unit mean density $(\rho=1)$, this process is generated within a hypercubic simulation box of fixed volume $V$ under periodic boundary conditions by a twostep procedure. First, we choose a random number $N$ from the Poisson distribution (6) with intensity or mean $\rho V=V$ and then uniformly place $N$ points in the simulation box.

\section{Equilibrium packings}

We also consider equilibrium packings of identical sphere (Gibbs hard-sphere processes) across the first three space dimensions. For $d=2$ and $d=3$, we examine disordered states that lie along the stable liquid branch $[5,29]$ as well as disordered states in one dimension, all of which are nonhyperuniform. We generate such equilibrium packings using the well-established Metropolis numerical scheme $[5,29]$. All configurations that we generate are far 

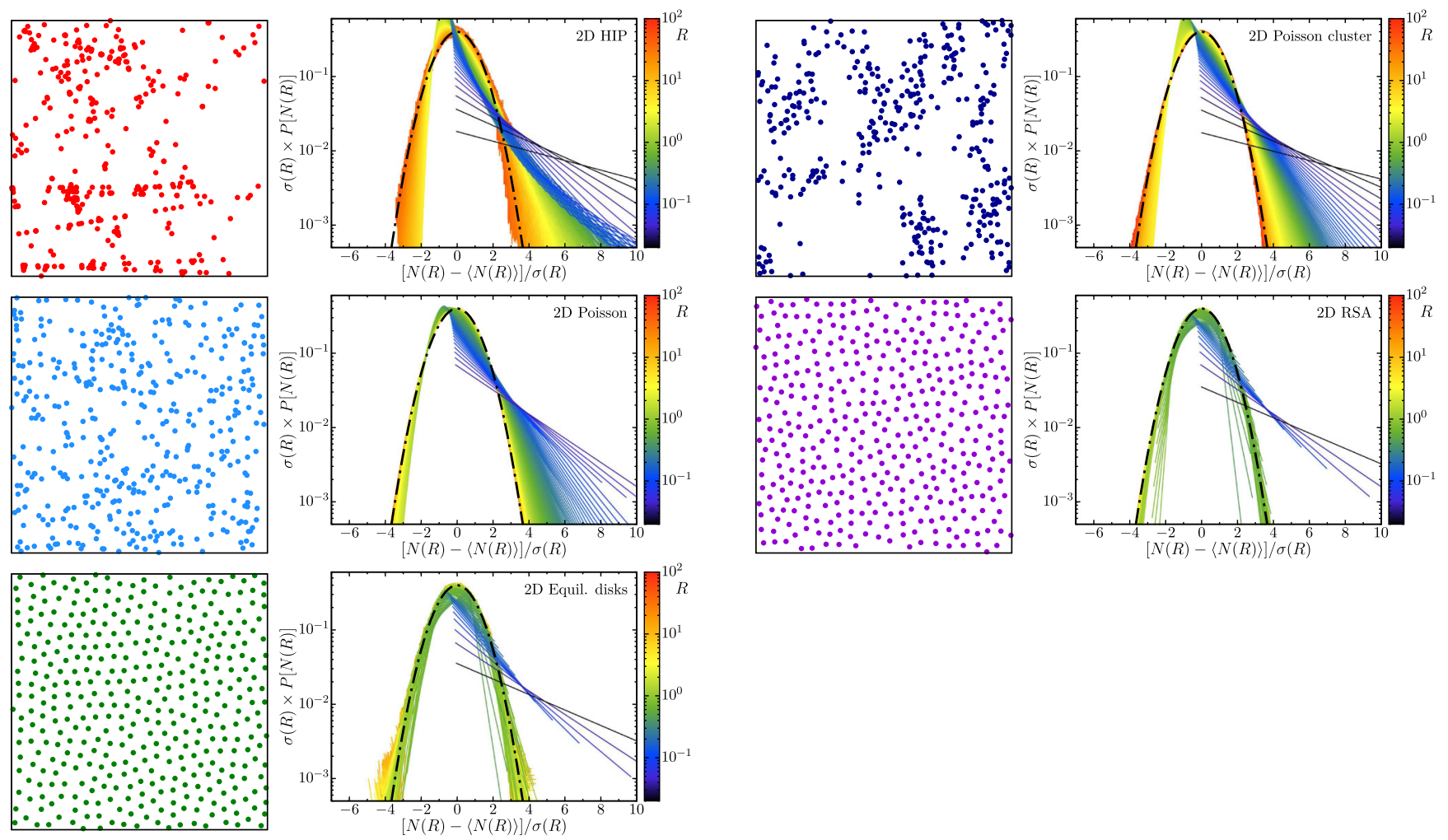

FIG. 3. Representative images of configurations of each of the five $2 \mathrm{D}$ nonhyperuniform models, beginning with the most nonhyperuniform one (HIP) down to one that has the smallest structure factor at the origin (equilibrium disks). The corresponding standardized probability distributions for different window radii are also included; the deepest blue color (darkest shade) corresponds to zero radius, and the deep orange color corresponds to the largest radius considered.
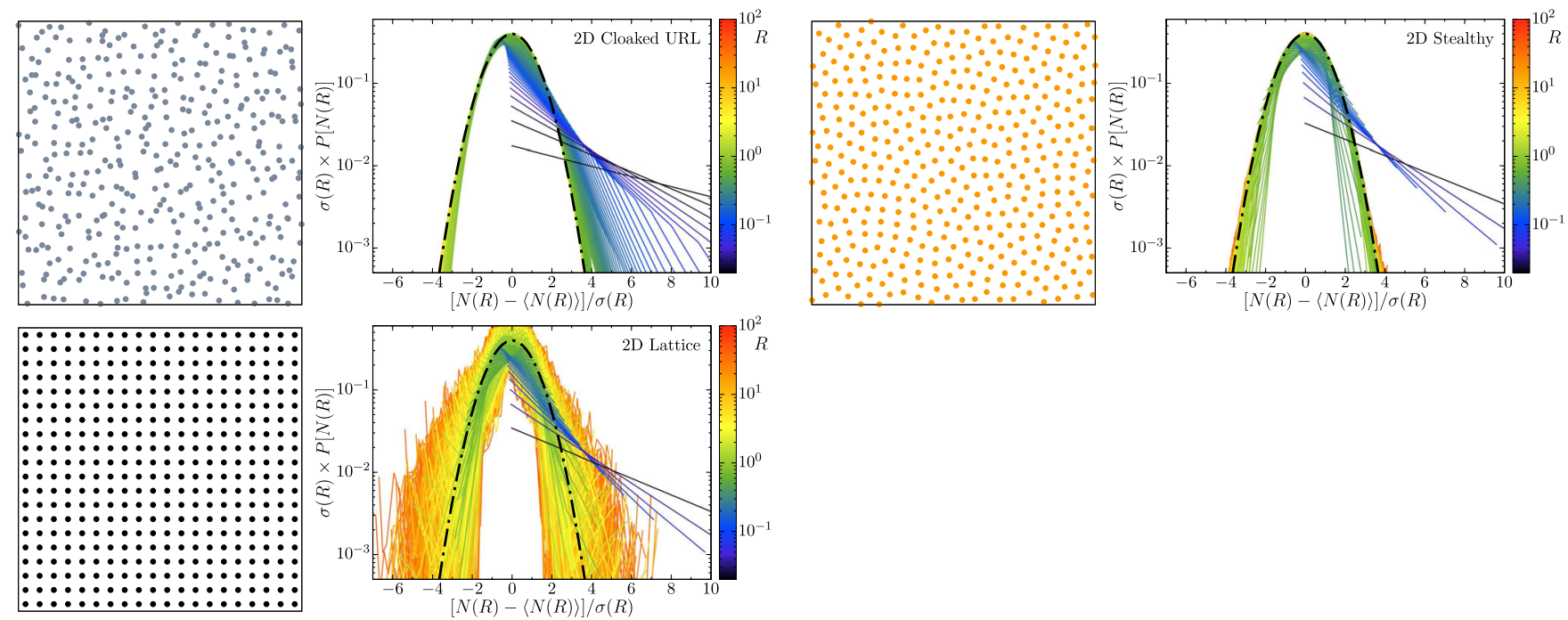

FIG. 4. Representative images of configurations of each of the three 2D hyperuniform models and their corresponding standardized probability distributions, where the deepest blue color (darkest shade) corresponds to zero radius and the deep orange color corresponds to the largest considered radius. The color code reveals differences between the results in this figure compared to the corresponding nonhyperuniform cases shown in Fig. 3. 
away from jamming points, and hence, they are all nonhyperuniform with bounded $S(0)$ (see Table I).

\section{Random sequential addition packings}

The random sequential addition (RSA) process is a timedependent (nonequilibrium) procedure that generates disordered sphere packings in $\mathbb{R}^{d}[39,75,92-95]$. Starting with an empty but large volume in $\mathbb{R}^{d}$, the RSA process is produced by randomly, irreversibly, and sequentially placing nonoverlapping spheres into the volume. This procedure is repeated for ever-increasing volumes; then, an appropriate infinite-volume limit is obtained. In practice, hard spheres are randomly and sequentially placed into a large fundamental cell under periodic boundary conditions and subjected to a nonoverlap constraint: If a new sphere does not overlap with any existing spheres, it will be added to the configuration; otherwise, the attempt is discarded. One can stop the addition process at any time $t$, obtaining RSA configurations with a range of packing fractions $\phi(t)$ up to the maximal saturation value $\phi_{s} \equiv \phi(\infty)$, which imposes a bounded-hole property [86]. For identical spheres, which we consider here, $\phi_{s} \approx 0.74,0.55$, and 0.38 for $d=1,2$, and 3 , respectively [38,39,92,94,95]. The pair correlation function $g_{2}(r)$ is known exactly only in one dimension [96]. The structure factors at the saturation states across dimensions have been determined numerically $[38,39]$. These results reveal that saturated RSA packings are nonhyperuniform, even if the values of $S(0)$ are relatively small (see Table I).

\section{Poisson cluster process}

The Poisson cluster process is an example of a strongly clustering point process with large density fluctuations on large length scales, i.e., with a large but finite value of $S(0)$, and hence is a nonhyperuniform system that is far from being hyperuniform. The construction of the cluster process starts from a homogeneous Poisson point process of intensity $\rho_{p}$ [24]. Each point of the Poisson point process is the center of a cluster of points. The number of points in each cluster is independent and follows a Poisson distribution with mean value $c$. In our specific model, the positions of the points relative to the center of the cluster follow an isotropic Gaussian distribution with standard deviation $r_{0}$, which can be regarded as the characteristic length scale of a single cluster. This model is also known as a (modified) Thomas point process, which is an example of a Neyman-Scott process $[49,50]$. In the infinite-volume limit, the pair correlation function in $\mathbb{R}^{d}$ is exactly given by [50]

$$
g_{2}(r)=1+\frac{c}{\rho\left(4 \pi r_{0}^{2}\right)^{d / 2}} e^{-\frac{r^{2}}{4 r_{0}^{2}}}
$$

Thus, the corresponding structure factor for any $d$ is given by

$$
S(k)=1+c e^{-k^{2} r_{0}^{2}},
$$

and hence such processes are nonhyperuniform and superPoissonian with $S(0)=1+c$. To simulate the process, which is straightforward, we use periodic boundary conditions and the following parameters across the first three space dimensions: $r_{0} \rho^{1 / d}=1$ and unit number density $\rho=$ $\rho_{p} c=1$ such that $\rho_{p}=0.1$ and $c=10$. For such parameters, $S(0)=11$ across dimensions (see Table I).

\section{Hyperplane intersection process}

The hyperplane intersection process (HIP) is hyperfluctuating [16]; i.e., its number variance scales faster than the volume of the observation window, and $\lim _{k \rightarrow 0} S(k)=$ $\infty$ [26,97]. This antihyperuniform and super-Poissonian point process is defined as the vertices (i.e., intersections of $d$ hyperplanes) of a Poisson hyperplane process - that is, of randomly and independently distributed hyperplanes $[49,98]$. In the infinite-volume limit, the pair correlation function in $\mathbb{R}^{d}$ for any $d \geq 2$ is exactly given by [26]

$g_{2}(r)=1+\sum_{k=1}^{d-1}\left(\begin{array}{c}d-1 \\ k\end{array}\right)\left(\frac{\omega_{d-k}}{\omega_{d}}\right)^{2}\left(\frac{d \omega_{d}}{\omega_{d-1}}\right)^{k} \frac{1}{(s r)^{k}}$,

where $s$ is the specific surface of the hyperplane process (i.e., the expected surface area of the hyperplanes per unit volume) and $\omega_{d}$ denotes the volume of a $d$-dimensional sphere of unit radius. The number density $\rho$ is determined by the specific surface area $s$ of the Poisson hyperplane process (which is the only parameter of the isotropic HIP):

$$
\rho=\omega_{d}\left(\frac{\omega_{d-1}}{d \omega_{d}}\right)^{d} s^{d} .
$$

According to Eq. (77), because $\alpha=1$ for any $d$, the number variance has the large- $R$ scaling $\sigma^{2}(R) \sim R^{2 d-1}$. Clearly, this process does not exist for $d=1$. To simulate this process, we cannot employ periodic boundary conditions; rather, we circumscribe the cubic simulation box by a hypersphere and then generate intersecting hyperplanes that are Poisson distributed [97]. The orientation of the hyperplanes is uniformly distributed on the unit sphere, and the distance of the hyperplanes to the center of the simulation box is uniformly distributed between zero and the radius of the circumsphere. The point process at unit number density is then simulated by computing all intersections of hyperplanes (within the circumsphere).

\section{B. Hyperuniform processes}

\section{Hypercubic lattice}

Interestingly, the problem of determining number fluctuations in lattices has deep connections to number theory, including Gauss's circle problem [99] and its 
generalizations [16] as well as the Epstein zeta function [100], which is directly related to the minimization of the number variance $[13,16,90]$. All periodic point patterns in $\mathbb{R}^{d}$, including Bravais lattices, are hyperuniform of class I $[13,16,90]$. The hyperuniformity concept enables one to rank order lattices and other periodic point patterns according to the degree to which they suppress large-scale density fluctuations as defined by the number variance $[13,16,90]$.

For the purposes of this investigation, it is sufficient to consider the higher-order fluctuations of the hypercubic lattice $\mathbb{Z}^{d}$ defined by

$$
\mathbb{Z}^{d}=\left\{\left(x_{1}, \ldots, x_{d}\right): x_{i} \in \mathbb{Z}\right\} \quad \text { for } d \geq 1,
$$

where $\mathbb{Z}$ is the set of integers $(\ldots-3,-2,-1,0,1,2,3 \ldots)$ and $x_{1}, \ldots, x_{d}$ denote the components of a lattice vector.

\section{Uniformly randomized lattice}

It is well known that if the sites of a lattice are stochastically displaced by certain finite distances, the scattering intensity (structure factor) inherits the Bragg peaks (long-range order) of the original lattice, in addition to a diffuse contribution. It has recently been demonstrated that these Bragg peaks can be hidden in the scattering pattern for certain independent and identically distributed perturbations [61]. We refer to this protocol as the uniformly randomized lattice (URL) model [61]. The underlying long-range order can be "cloaked," under certain conditions, in the sense that it cannot be reconstructed from the pair-correlation function alone. Here, we generate the URL model using the hypercubic lattice $\mathbb{Z}^{d}$ and displace each lattice point by a random vector that is uniformly distributed in a rescaled fundamental cell of the lattice with $a F \equiv[-a / 2, a / 2)^{d}$. The constant $a$ controls the strength of perturbations. Counterintuitively, the long-range order suddenly disappears at certain discrete values of $a$ and reemerges for stronger perturbations. Here, we cloak the Bragg peaks of $\mathbb{Z}^{d}$ using the special value $a=1$. Such cloaked URLs are hyperuniform such that $S(k) \sim k^{2}$ in the limit $k \rightarrow 0$ and hence are of class I [see Eq. (76)].

\section{Stealthy hyperuniform process}

Stealthy hyperuniform processes are defined by a structure factor that vanishes in a spherical region around the origin, i.e., $S(\mathbf{k})=0$ for $0<|\mathbf{k}| \leq K$. Such point processes are hyperuniform of class I; see Eq. (76). A powerful procedure that enables one to generate high-fidelity stealthy hyperuniform point patterns is the collective-coordinate optimization technique [101-106]. This optimization methodology involves finding the highly degenerate ground states of a class of bounded pair potentials with compact support in Fourier space, which are stealthy and hyperuniform by construction. The control parameter $\chi$ is a dimensionless measure of the ratio of constrained degrees of freedom (i.e., wave vectors contained within the cutoff wave number $K$ ) to the total degrees of freedom (approximately $d N$ ) in such an optimization procedure. A point configuration with a small value of $\chi$ (relatively unconstrained) is disordered, and as $\chi$ increases, the short-range order increases within a disordered regime $(\chi<1 / 2$ for $d=2$ and $d=3$ ) [105]. For $d=1$, stealthy hyperuniform states can be disordered for $\chi<1 / 3$ [106]. Here, we use the collective-coordinate procedure to generate "entropically favored" disordered stealthy point processes by first performing molecular dynamics simulations at sufficiently low temperatures and then minimizing the energy to obtain ground states with exquisite accuracy [106]. Importantly, stealthy states possess the bounded-hole property [86,88], and hence, as discussed in Sec. III C, $P[N(R)=0]=0$ for $R>R_{\max }(\chi)$, where $R_{\max }(\chi)$ is the radius of the largest hole in space dimension $d$, which depends on the control parameter $\chi$.

\section{Link to other physical systems}

In Table II, we list a large number of physical systems $[7,16,24,26,29,41-47,49-70]$ that are related to our theoretical models, especially in regards to qualitatively similar values of $S(0)$. For this reason, we expect these related physical systems to also obey the aforementioned universality; i.e., their fluctuations are approximated well by the gamma distribution whenever a CLT is obeyed. The examples listed in Table II include two-phase media and random fields because the point configurations can be mapped to these systems by decorating each point with given shapes or kernels, respectively $[58,69,107,108]$. These mapped systems inherit the properties of the underlying point process. Thus, many of our results are expected to qualitatively extend to an even broader class of materials than many-particle systems. Moreover, note that our results for the simple cubic lattice qualitatively extend to general lattices or crystals. We also note that while we studied randomly perturbed crystals, these results are expected to be qualitatively similar to other low-temperature imperfect crystal phases, including those containing vacancies and phonons [61-66].

\section{GAUSSIAN DISTANCE METRIC}

As noted in the Introduction, we are interested in ascertaining how large $R$ must be such that $P[N(R)]$ is well approximated by the normal distribution. There are several candidate distance metrics we have considered that could be used to quantify proximity to the Gaussian distribution (beyond the skewness and excess kurtosis). One possible distance metric that we considered is the Kolmogorov-Smirnov test statistic [109]. While it is statistically robust, we found it to be too insensitive for our purposes. The Kullback-Leibler divergence (also 
called the relative entropy) is a well-known measure of the difference between probability distributions [110]. However, it is not well defined for comparing a discrete to a continuous distribution; see the SM [81] for details.

A recent study that considered distance metrics between a pair of general functions that depend on $d$-dimensional vectors was based on the integrated squared difference in $\mathbb{R}^{d}$, i.e., an $L_{2}$ distance metric [111]. These authors also found that the Kullback-Leibler divergence was not useful for their purposes. These findings motivated us to consider distance metrics based on the squared difference between the Gaussian and number distributions.

Here, we identify two different contributions to the distance between a number distribution $P[N(R)]$ and a Gaussian distribution: (1) deviations in the functional form of $P[N(R)]$ and (2) the discreteness of $N(R)$ that can only approximate the continuous Gaussian distribution. We find that the second contribution is essentially determined by the value of the number variance $\sigma^{2}(R)$; i.e., the contribution is smaller for larger values of $\sigma^{2}(R)$ for the following reason. Consider the standardized random variable $[N(R)-\langle N(R)\rangle] / \sigma(R)$ whose discrete probability distribution approximates the continuous normal density. Then, the bin width of the probability distribution is given by $1 / \sigma(R)$ and converges to zero for $\sigma(R) \rightarrow \infty$.

Moreover, we find that the weight of the two contributions (relative to each other) strongly depends on the representation of the number distribution, e.g., via the characteristic function (Fourier representation) [109] or via direct space representations either in discrete or continuous forms. In fact, the choice of representation can virtually reverse the order of the distance metrics for our point processes. For example, a strong contribution (2) may result in a lower value of the distance metric for the highly skewed distribution of the HIP than for the Poisson process. Because contribution (2) of the discreteness of $P[N(R)]$ is already essentially given by $\sigma^{2}(R)$, here we choose a representation that focuses on deviations in the functional form of $P[N(R)]$ from that of a Gaussian random variable.

Therefore, we define an integer-valued random variable $G(R)$, whose probability distribution $P_{G(R)}$ is proportional to a Gaussian distribution with the same first and second moments as our number distribution at radius $R$. We introduce a type of $L_{2}$ distance metric, denoted by $l_{2}(R)$, which is a Gaussian distance metric that employs the cumulative distribution function:

$$
l_{2}(R) \equiv\left[\frac{1}{\sigma(R)} \sum_{n=0}^{\infty}\left|F_{G}(n)-F_{N}(n)\right|^{2}\right]^{1 / 2},
$$

where $F_{G}(n)$ is the cumulative distribution function of $G(R)$, i.e.,

$$
F_{G}(n)=\sum_{m=0}^{n} P_{G(R)}[G(R)=m]
$$

and $F_{N}(n)$ is the cumulative probability distribution of $N(R)$, i.e.,

$$
F_{N}(n)=\sum_{m=0}^{n} P[N(R)=m]
$$

Note that the series of the squared differences in Eq. (81) is scaled by $1 / \sigma(R)$ because, for a Gaussian distribution, the range of values for which $F_{G} \in[\varepsilon, 1-\varepsilon]$ is proportional to $\sigma(R)$. If a particular number distribution converges (sufficiently fast) to a Gaussian distribution, $l_{2}(R)$ will tend to zero. In summary, the Gaussian distance metric (81) is designed to be sensitive to small deviations in the distribution functions and, at the same time, robust against statistical fluctuations.

\section{SAMPLING OF MOMENTS AND NUMBER DISTRIBUTION}

We sample number fluctuations within a spherical window of radius $R$ for all models using a two-step procedure. First, we randomly place an observation window in the sample (using a uniform distribution for its center). Second, we determine the number of points $N(R)$ within the observation window using periodic boundary conditions, except for the HIP, as described in Sec. IV A 5. To reduce computational resources, we use the same centers for all radii that we consider. Relevant simulation parameters and properties for each of the models described above across dimensions are listed in Table I.

Thus, we empirically determine the probability distribution $P[N(R)]$ and compute the mean value, variance, skewness, and excess kurtosis. We determine the first four central moments using the unbiased estimators from Ref. [112]. Finally, we compute the $l_{2}$ distance metric, as described above. A source of systematic errors, which must be avoided for both the moments and distance measures, can arise when the number of observation windows $N_{\text {window }}$ per sample is too large. This effect can cause the distance metric $l_{2}(R)$ to artificially increase again for large radii. Therefore, we have used between 1 and $10^{4}$ observation windows per sample, depending on the system size and computational cost, so that the systematic error is either avoided completely, or it is smaller than the effects caused by statistical fluctuations. As a rule of thumb, we choose $N_{\text {window }}$ such that the volume fraction of the union of all observation windows of the largest window radius $R_{\max }$ in one sample should not exceed $50 \%$, i.e., $1-\exp \left[-N_{\text {window }} v_{1}\left(R_{\max }\right) / V\right]<0.5$, where $V$ is the volume of a single sample. This criterion is based on the exact formula for the volume fraction of overlapping spheres [49]. Our estimators for $l_{2}(R)$ are statistically robust for 
values that are larger than the inverse of the square root of the total number $n$ of observation windows, where $n=N_{\text {window }} \times N_{c}$, with $N_{c}$ being the number of configurations. For a finite number of samples, we empirically find that $l_{2}(R)$ typically cannot be smaller than approximately $O(1 / \sqrt{n})$. Therefore, we apply a data cut and only consider radii up to

$$
R_{\text {cut }} \equiv \min _{R>3}\left\{R: l_{2}(R)<1 / \sqrt{n}\right\} .
$$

We apply the same data cut to the skewness and excess kurtosis [113]. We show all data without the data cut in the SM [81]. All simulated data are available at a Zenodo repository [114].

Another obvious source of systematic errors can arise when the size of the sampling window is not much smaller than the size of the simulation box with a fixed number of particles, i.e., when canonical ensembles are employed. It is well known that such finite-size effects lead to an underestimation of the local number variance $\sigma_{\text {finite }}^{2}(R)$ when compared to its value in the thermodynamic limit. An empirical formula to estimate the first-order correction to the thermodynamic limit [17] shows that the error term is proportional to $S(0)$ (because there are larger fluctuations in the number of points per simulation box). Importantly, this implies that hyperuniform models, defined by $\lim _{k \rightarrow 0} S(k)=0$, are more robust against such finite-size effects than nonhyperuniform models.

\section{RESULTS}

We describe the results that we obtained for the second, third, and fourth cumulants, $\sigma^{2}(R), \gamma_{1}(R)$, and $\gamma_{2}(R)$, as a function of the window radius $R$ for all models across the first three space dimensions at unit number density $(\rho=1)$, as well as the corresponding full probability distributions $P[N(R)]$ and the Gaussian distance metric $l_{2}(R)$. A testament to the high precision of the data is the excellent agreement with rigorous bounds for these quantities for general cases, as well as with exact results for the cases of packings for certain $R$ reported in Sec. III (see the SM [81] for details).

\section{A. Cumulants $\sigma^{2}(R), \gamma_{1}(R)$, and $\gamma_{2}(R)$ for the models across dimensions}

Figure 2 shows the second, third, and fourth cumulants as a function of $R$ versus the window radius $R$ for all models for $d=1, d=2$, and $d=3$. The large- $R$ asymptotic behavior of the variance is determined by the structure factor at the origin, $S(0)$ [13]. As expected, the scaled number variance $\sigma^{2}(R) / v_{1}(R)$ grows with $R$ for the HIP process for two-dimensional (2D) and three-dimensional (3D) cases. For all other nonhyperuniform models, $\sigma^{2}(R) / v_{1}(R)$ asymptotes to a constant for large $R$ in all dimensions. Of course, this scaled variance decreases with $R$ for the three hyperuniform models (hypercubic lattice, URL, and stealthy systems).

For $d \geq 2$, we have found, via analyses given in the SM [81] and immediately below, that the skewness $\gamma_{1}(R)$ and excess kurtosis $\gamma_{2}(R)$ for our disordered hyperuniform systems vanish faster with increasing $R$ than those for nonhyperuniform systems. Among all models studied, the quantities $\gamma_{1}(R)$ and $\gamma_{2}(R)$ vanish slowest for the antihyperuniform HIP models for $d \geq 2$. The specific decay rates for all models are described below.

It is noteworthy that one-dimensional systems can present fluctuation anomalies not present in higher dimensions. For example, in the case of the integer lattice, the random variable $N(R)$ can only take, at most, two values for any $R$, which of course is abnormally non-Gaussian. While the hypercubic lattice for $d \geq 2$ never achieves a CLT (as discussed below), the variance is considerably broader than that for $d=1$. Another anomalous category is class I hyperuniform systems, which have bounded variance for $d=1$ [see Eq. (76)], and thus no such hyperuniform point process can obey a CLT because the standardized distribution cannot converge to a continuous distribution as $R$ becomes large. This case is clearly borne out by the distance metric plots, shown in Fig. 5 and in Fig. S8 of the SM [81], for both the 1D disordered stealthy point process and the integer lattice.

For the Poisson and super-Poissonian models (cluster and HIP) across dimensions, both the skewness and excess kurtosis decay monotonically; see Fig. 2. By contrast, the
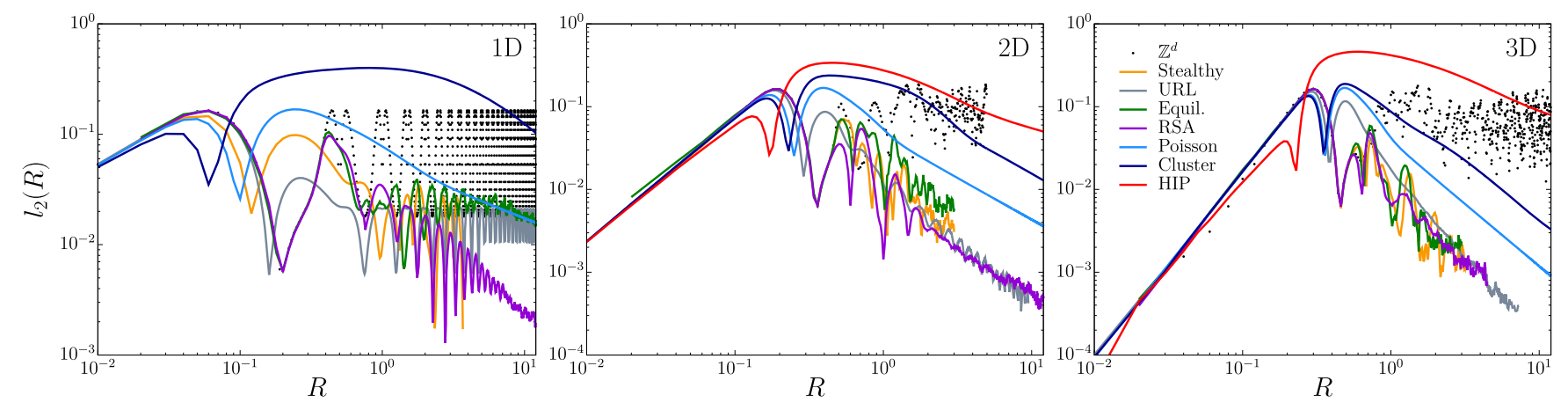

FIG. 5. Gaussian distance metric $l_{2}(R)$ versus the window radius $R$ for all considered models across the first three space dimensions. 
skewness and excess kurtosis oscillate about zero for the lattice, stealthy, equilibrium, and RSA systems across all dimensions because all of them exhibit at least short-range order. It should not go unnoticed how the oscillations in $\gamma_{1}(R)$ and $\gamma_{2}(R)$ are related to short- or long-range order at the level of three- and four-body correlation functions, $g_{3}$ and $g_{4}$, as can be seen from the explicit formulas (22) and (23) for the skewness and excess kurtosis. In the instances of RSA and equilibrium packings, oscillations in $\gamma_{1}(R)$ and $\gamma_{2}(R)$ arise from strong short-range order exhibited by $g_{3}$ and $g_{4}$.

The disordered hyperuniform systems that we consider (stealthy and URL point processes) have extraordinary number fluctuation behaviors. For 2D and 3D stealthy systems, both $\gamma_{1}(R)$ and $\gamma_{2}(R)$ strongly oscillate about zero; see Fig. 2. These cases suggest that, at the level of $g_{3}$, stealthy systems, counterintuitively, exhibit significant ordering on much larger length scales than the short-range order seen in the pair correlation function [105]. For 1D stealthy systems, $\gamma_{1}(R)$ and $\gamma_{2}(R)$ show even stronger oscillations than their higher-dimensional counterparts, indeed reflecting possible long-range order present in $g_{3}$ and $g_{4}$. It is remarkable that the skewness and excess kurtosis can detect such anomalous long-range order, which would not be expected based solely on the behavior of the pair correlation function. Another model that supports the capacity of $\gamma_{1}(R)$ and $\gamma_{2}(R)$ to detect unusual long-range order is the cloaked URL model, which, at the level of the pair correlation function, is considered to be highly disordered. Whereas $\gamma_{1}(R)$ for this model is a monotonically decreasing function of $R, \gamma_{2}(R)$ exhibits oscillations. This result is entirely consistent with the fact that the periodicity of the underlying lattice is completely hidden at the level of the three-point correlation function but manifests itself for the first time in $g_{4}$ [61].

\section{B. Number distributions for the models across dimensions}

Figures 3 and 4 show representative configurations of all of the 2D models and their corresponding standardized number distributions. (We provide corresponding figures for all 1D and 3D models in the SM [81]). Except for hypercubic lattices for any $d$ and 1D hyperuniform systems of class I, all of the considered models across dimensions obey a CLT. It is noteworthy that for all models, except the hypercubic lattices, the number distribution functions $P[N(R)]$ are unimodal, i.e., one with a single peak. (For small radii $R \lesssim 0.5$, the number distributions are monotonically decreasing and are still considered to be unimodal.) Recall that for all models, except the hypercubic lattice and the class I hyperuniform models in one dimension, the skewness and excess kurtosis tend to zero for large $R$ for all dimensions. For well-behaved unimodal distributions, such a vanishing of both $\gamma_{1}(R)$ and $\gamma_{2}(R)$ indicates a tendency to a CLT. Indeed, this tendency is confirmed by visual inspection of our corresponding evaluations of the number distributions for all dimensions; see Figs. 3 and 4. Our conclusions about the tendency to CLTs for sufficiently large $R$ are further confirmed by our evaluations of the Gaussian distance metric $l_{2}(R)$ for all models across dimensions, which are plotted in Fig. 5. For $d=2$ and $d=3$, disordered hyperuniform point processes tend to be better approximated by the normal distribution than their nonhyperuniform counterparts at a given large value of $R$ based upon the different scalings of $l_{2}(R)$, as shown below. From Fig. 5 and Fig. S8 of the SM [81], one can ascertain, for each model, a radius $R_{0}$ above which the distribution can be deemed to be approximately Gaussian; i.e., $l_{2}(R)$ is below some threshold for $R>R_{0}$, implying that it is approximately determined by only the mean and the variance. Typically, we find that $R_{0}$ is orders of magnitude smaller for our hyperuniform and standard nonhyperuniform models than for our super-Poissonian models.

Our results clearly show that the hypercubic lattices across dimensions do not obey a CLT; see Figs. 2, 4, and 5. This is due to the fact that lattice points, in general, are "rigid" in the sense that fluctuations in $N(R)$ are always stringently bounded from below and above for any value of $R$ (as discussed in Sec. III C) because of their inherent longrange order. Hence, $P[N(R)]$ is highly sensitive to the value of $R$; thus, both $\gamma_{1}(R)$ and $\gamma_{2}(R)$ rapidly oscillate around zero, but the amplitudes of the oscillations do not vanish as $R$ increases. It is already well established that the local variance $\sigma^{2}(R)$ of lattices exhibits such rapid oscillations; see Ref. [13] and references therein. Because of the rapid oscillations in $\gamma_{1}(R), \gamma_{2}(R)$, and $l_{2}(R)$ for hypercubic lattices, we represent the data by points instead of curves, which would require interpolation between the data points. Note that the corresponding distance metrics $l_{2}(R)$ are bounded from below for any value of $R$. These observations are consistent with the visual inspections of the number distribution shown in Fig. 4 for $d=2$ and those for $d=1$ and $d=3$ given in the SM [81].

\section{Universal gamma-distribution approximation for all models obeying a CLT}

We have studied a variety of well-known, closed-form, probability distributions to determine those that best approximate the actual distributions for finite $R$ for all of our models. Remarkably, we have ascertained that the gamma distribution provides a good approximation to the number distribution $P[N(R)]$ for all models that obey a CLT across all dimensions for intermediate to large values of $R$. (Recall that the hypercubic lattices and 1D hyperuniform systems do not obey a CLT.) The gamma distribution is defined by

$$
P[N(R)=m]=\frac{1}{\Gamma(k) \theta^{k}} m^{k-1} e^{-m / \theta},
$$



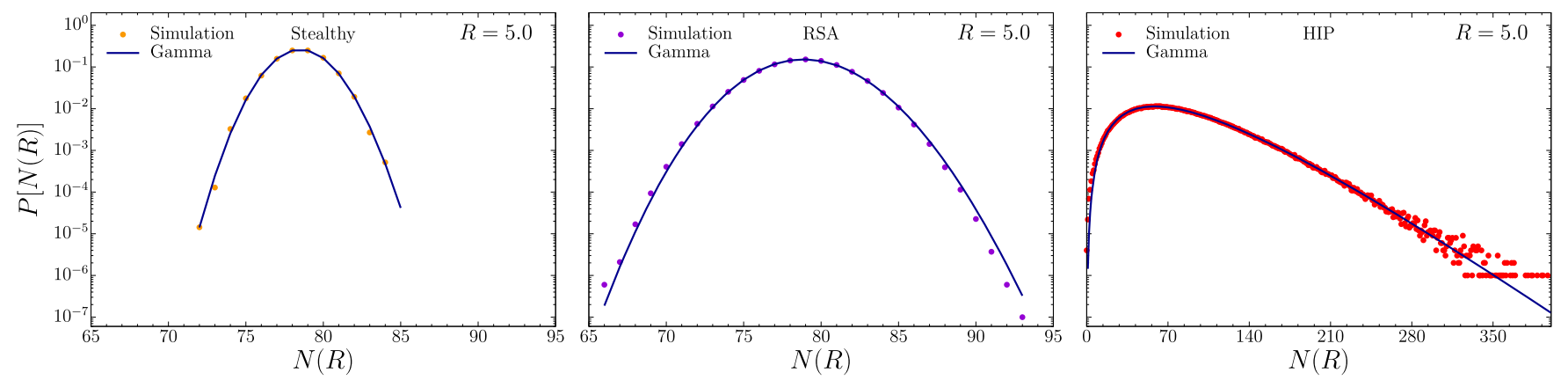

FIG. 6. Comparison of simulation data for the probability distributions $P[N(R)]$ with $R=5$ for three $2 \mathrm{D}$ models (stealthy hyperuniform, RSA, and HIP) to corresponding gamma-distribution approximations with the same mean value and variance. The gamma distribution provides good approximations for all models that obey a CLT across the first three dimensions considered, including the ones not shown here.

where $k$ and $\theta$ are shape and scale parameters, respectively, which are related to the mean and variance of $P[N(R)]$ as follows:

$$
\begin{gathered}
\langle N(R)\rangle=k \theta, \\
\sigma^{2}(R)=k \theta^{2},
\end{gathered}
$$

implying that $k=\langle N(R)\rangle^{2} / \sigma^{2}(R)$ and $\theta=\sigma^{2}(R) /\langle N(R)\rangle$. It immediately follows that the associated skewness and excess kurtosis can be expressed simply in terms of the mean and number variance, yielding

$$
\begin{gathered}
\gamma_{1}(R)=\frac{2}{\sqrt{k}}=2\left[\frac{\sigma^{2}(R)}{\langle N(R)\rangle^{2}}\right]^{1 / 2}, \\
\gamma_{2}(R)=\frac{6}{k}=6\left[\frac{\sigma^{2}(R)}{\langle N(R)\rangle^{2}}\right] .
\end{gathered}
$$

Figure 6 compares the gamma-distribution approximations to simulation data for a representative disordered hyperuniform model (stealthy), a standard nonhyperuniform model (RSA), and an antihyperuniform model (HIP) in two dimensions for $R=5$. Visual inspection reveals that the gamma distribution provides a good approximation in each case. Similar good agreement between the gammadistribution approximation and simulation data was found for other models (not shown in Fig. 6) obeying a CLT across dimensions, as discussed in the SM [81]. The fact that the number distributions for the majority of our models closely follow a gamma distribution is reasonable, since both of them are unimodal, supported in the interval $[0, \infty)$, and they have (on average) positive skewness and excess kurtosis, i.e., right skewness and heavier tails than Gaussian.

Importantly, the approximation of $P[N(R)]$ by a gamma distribution enables us to estimate the large- $R$ scalings of $\gamma_{1}(R)$ and $\gamma_{2}(R)$ for all models across dimensions that obey a CLT. Specifically, we find $\gamma_{1}(R) \sim R^{-1 / 2}$ and $\gamma_{2}(R) \sim$ $R^{-1}$ for the antihyperuniform HIP, $\gamma_{1}(R) \sim R^{-d / 2}$ and
$\gamma_{2}(R) \sim R^{-d}$ for standard nonhyperuniform models, and $\gamma_{1}(R) \sim R^{-(d+1) / 2}$ and $\gamma_{2}(R) \sim R^{-(d+1)}$ for the hyperuniform models. Table III summarizes these scaling behaviors. The fact that the excess kurtosis decays to zero faster than the skewness for any model that obeys a CLT, whether hyperuniform or not, implies that the dominant asymptotic correction of the gamma distribution to a CLT for large $R$ is determined by the skewness; see the Appendix for a proof. It is noteworthy that these predictions based on the gamma distribution are consistent with numerical findings for all nonhyperuniform systems and the antihyperuniform HIP using an independent method that employs certain running averages of $\gamma_{1}(R)$ and $\gamma_{2}(R)$; see the SM [81] for details. Moreover, for 2D and 3D disordered hyperuniform (stealthy and URL) models, the predictions from the gamma-distribution approximations are also consistent with the observed scalings of the skewness. Because of the strong oscillations in the excess kurtosis for the 2D and 3D stealthy and URL processes described above, it is numerically difficult to definitively determine their scalings from the running-average method. It should not go unnoticed that the exact formulas for the skewness and excess kurtosis, Eqs. (34) and (35), respectively, of the Poisson distribution are consistent with the scalings predicted by the gamma-distribution approximation, lending additional validity to the latter. Furthermore, we have found that for all models across dimensions that obey a CLT, the runningaverage procedure yields scalings for $l_{2}(R)$ that agree with

TABLE III. Large- $R$ asymptotic scalings of $\sigma^{2}(R), \gamma_{1}(R)$, $\gamma_{2}(R)$, and $l_{2}(R)$ for all of our models across dimensions that obey a CLT, as obtained by the approximation of $P[N(R)]$ by the gamma distribution function for each model for any $d$.

\begin{tabular}{lccc}
\hline \hline & $\begin{array}{c}\text { Stealthy } \\
\text { Descriptor }\end{array}$ & $\begin{array}{c}\text { Cluster, Poisson, RSA, } \\
\text { and URL }\end{array}$ & and equilibrium \\
\hline$\sigma^{2}(R)$ & $R^{(d-1)}$ & $R^{d}$ & $R^{2 d-1}$ \\
$\gamma_{1}(R)$ & $R^{-(d+1) / 2}$ & $R^{-d / 2}$ & $R^{-1 / 2}$ \\
$\gamma_{2}(R)$ & $R^{-(d+1)}$ & $R^{-d}$ & $R^{-1}$ \\
$l_{2}(R)$ & $R^{-(d+1) / 2}$ & $R^{-d / 2}$ & $R^{-1 / 2}$ \\
\hline \hline
\end{tabular}



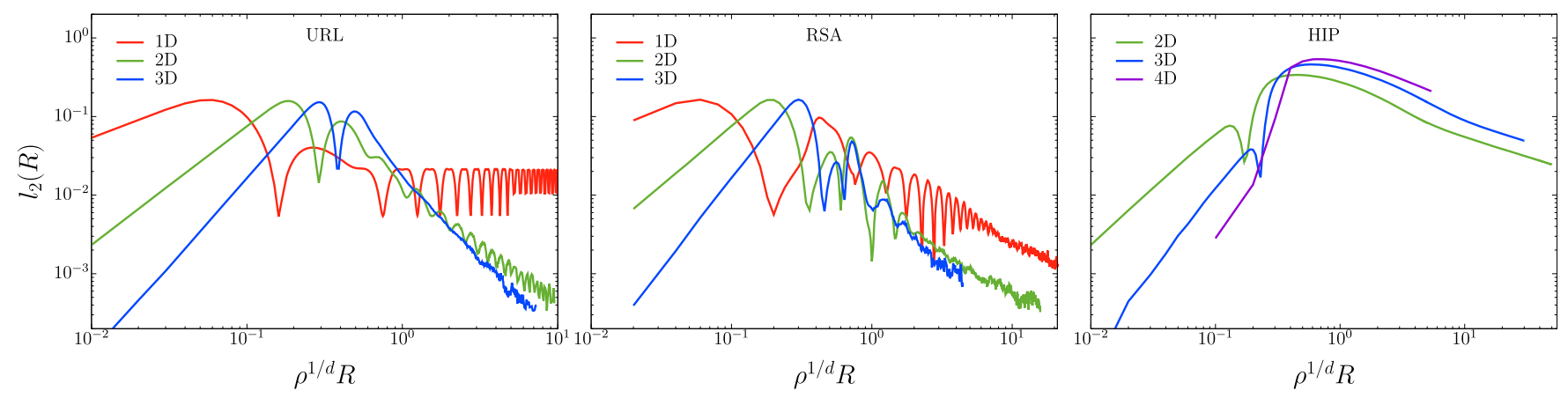

FIG. 7. Gaussian distance metric $l_{2}(R)$ versus $R$ for a representative disordered hyperuniform model (URL), a sub-Poisson nonhyperuniform model (RSA), and an antihyperuniform model (HIP) across the first three space dimensions, respectively.

the corresponding gamma-distribution approximations (see the SM [81]), which are identical to the scalings for the skewness $\gamma_{1}(R)$. The proof that $l_{2}(R)$ has exactly the same scaling as $\gamma_{1}(R)$ for the gamma distribution is given in the Appendix. Furthermore, our approximations of $P[N(R)]$ by gamma distributions are also consistent with CLT behaviors since they converge to the Gaussian distribution in the limit of $k \rightarrow \infty$ (i.e., $R \rightarrow \infty$ ), as described in the Appendix.

According to Table III, convergence to a CLT is slowest for the HIP (proportional to $R^{-1 / 2}$ for $d \geq 2$ ), followed by the Poisson cluster process and the Poisson process. RSA and equilibrium packings have the same scaling behaviors as the Poisson cluster and Poisson processes but with smaller coefficients of proportionality. The convergence to a CLT is fastest for the disordered hyperuniform processes for $d=2$ and $d=3$ [115] since convergence to a CLT is inversely correlated with the variance, as predicted by the gamma distribution.

\section{Effect of dimensionality on the approach to a CLT}

For any particular $d$-dimensional model that eventually obeys a CLT, does the number distribution function $P[N(R)]$ tend to Gaussian-like behavior faster as the space dimension $d$ increases? As noted in the Introduction, this question can be answered by appealing to the decorrelation principle [72], which states that unconstrained correlations in disordered packings that exist in low dimensions vanish as $d$ tends to infinity, and all higher-order correlation functions $g_{n}$ for $n \geq 3$ may be expressed in terms of the number density $\rho$ and pair correlation function $g_{2}$. The decorrelation principle begins to manifest itself in low dimensions for disordered packings $[38,74,75]$ as well as for other disordered systems with strongly repulsively interacting particles, including fermionic [76] and Gaussian-core point processes [77]. We know that the number distribution function $P[N(R)]$ generally involves certain integrals over all of the $n$-body correlation functions. Therefore, the decorrelation principle implies that for any $d$-dimensional model that decorrelates with $d, P[N(R)]$ increasingly becomes Gaussian-like as $d$ increases since the first and second moments, determined by $\rho$ and $g_{2}$, dominate the distribution. By the same token, for any model that correlates with increasing $d, P[N(R)]$ increasingly deviates from the normal distribution as $d$ increases, as verified by computing the pair correlation function given by Eq. (79). We have verified these broad conclusions for the models studied in this article. In Fig. 7, we plot the Gaussian distance metric $l_{2}(R)$ for a representative disordered hyperuniform model (URL), a sub-Poisson nonhyperuniform model (RSA), and an antihyperuniform model (HIP) across the first three space dimensions, respectively. (Recall that the URL obeys a CLT for $d \geq 2$.) As expected, we see that for both the URL and RSA models for a fixed value of $R$ (for $R>1$ ), $l_{2}(R)$ decreases with increasing $d$ (increasingly tending to Gaussian-like behavior) because of decorrelation, while for the HIP, $l_{2}(R)$ increases with increasing $d$ (moving away from Gaussian-like behavior) because it increasingly correlates with $d$. Importantly, when comparing fluctuations across dimensions, one must choose a meaningful length scale to make the window radius $R$ dimensionless. A simple and good choice is $\rho^{1 / d}$, which is inversely proportional to the mean nearest-neighbor distance in space dimension $d$, and it explains why the horizontal axes in each subfigure of Fig. 7 are $\rho^{1 / d} R$.

\section{CONCLUSIONS AND DISCUSSION}

Via theoretical methods and high-precision simulation studies, we accurately quantified the skewness $\gamma_{1}(R)$, excess kurtosis $\gamma_{2}(R)$, and the number distribution $P[N(R)]$ for eight different models of statistically homogeneous point processes in two and three dimensions: five nonhyperuniform models, one of which is antihyperuniform (HIP), and three hyperuniform models. Analogous models were also examined in one dimension, except for the HIP, which is not defined in this dimension. We validated our simulation results for $\gamma_{1}(R), \gamma_{2}(R)$, and $P[N(R)]$ for all models by showing that they are in excellent agreement with rigorous bounds and exact results that we derived for the applicable ranges of $R$. For all disordered hyperuniform models, our explicit general formulas for $\gamma_{1}(R)$ and $\gamma_{2}(R)$ in terms of $n$-body 
information [Eqs. (22) and (23)] enable us to infer the existence of a "hidden" type of long-range order that manifests itself for the first time at the three-body level or higher. Thus, the skewness and excess kurtosis have the capacity to detect anomalous long-range order that would not be expected based on the behavior of the pair correlation function alone. While the presence of threeand higher-body correlations can be inferred by the behaviors of the higher-order moments and $P[N(R)]$ (as we showed in the case of stealthy systems and the cloaked URL), such fluctuation information is still of importance even if the system is not characterized by strong correlations (e.g., gases and dilute liquids).

We have introduced a novel Gaussian distance metric $l_{2}(R)$ to ascertain the proximity of $P[N(R)]$ to the normal distribution for each model as a function of $R$. We have verified that $l_{2}(R)$ is a sensitive metric via numerical and theoretical methods. Since the distributions for all models (except the lattices) across dimensions are unimodal, the tendency to a CLT corresponds to the skewness and excess kurtosis simultaneously tending to zero. Almost all of the considered models across dimensions obey a CLT. We found that disordered hyperuniform point processes are better approximated by the normal distribution than their nonhyperuniform counterparts at a given large value of $R$. We proved that any 1D hyperuniform system of class I as well as the hypercubic lattice for any $d$ cannot obey a CLT. Similarly, a general lattice in any dimension cannot obey a CLT.

It is noteworthy that we discovered a type of universality in that the gamma distribution provides a good approximation to the number distribution $P[N(R)]$ for all models that obey a CLT across all dimensions for intermediate to large values of $R$, enabling us to estimate the large- $R$ scalings of $\gamma_{1}(R), \gamma_{2}(R)$, and $l_{2}(R)$. These predictions were corroborated by corresponding simulation results in almost all instances, as detailed in the SM [81]. It is only in the special cases of the excess kurtosis for 2D and 3D stealthy and URL processes where the running-average method was not reliable enough to definitively determine their scalings due to strong oscillations, and thus this represents a simulation challenge for future research. Among all models, the convergence to a CLT is generally fastest for the disordered hyperuniform processes in two and three dimensions, for reasons given above, such that $\gamma_{1}(R) \sim$ $l_{2}(R) \sim R^{-(d+1) / 2}$ and $\gamma_{2}(R) \sim R^{-(d+1)}$ for large $R$. The convergence to a CLT is slower for standard nonhyperuniform models such that $\gamma_{1}(R) \sim l_{2}(R) \sim R^{-d / 2}$ and $\gamma_{2}(R) \sim R^{-d}$. Not surprisingly, the convergence to a CLT is slowest for the anti-hyperuniform HIP model such that $\gamma_{1}(R) \sim l_{2}(R) \sim R^{-1 / 2}$ and $\gamma_{2}(R) \sim R^{-1}$. Using the decorrelation principle, we elucidated why any $d$-dimensional model that "decorrelates" or "correlates" with $d$ corresponds to a $P[N(R)]$ that increasingly moves toward or away from a CLT, respectively.
We emphasize that our work provides general principles that can be used to describe a wide class of real systems. First, the rigorous bounds on higher-order moments $\left[\gamma_{1}(R)\right.$ and $\left.\gamma_{2}(R)\right]$ and $P[N(R)]$, derived in Sec. III, are valid for general point configurations. Second, we found a universality in that the number distributions at intermediate values of $R$ are well approximated by the gamma distribution for all of our distinctly different models that obey a CLT, including stealthy hyperuniform as well as antihyperuniform systems. Moreover, the number distributions of a large number of other physical systems, summarized in Table II, should behave qualitatively similarly to those of our corresponding theoretical models. Since we found that models with similar values of $S(0)$ have similar fluctuation behaviors, we expect these related physical systems will obey the aforementioned universality, but this must be verified in future work. Note that for any system for which the universal approximation holds, $\rho$ and $\sigma^{2}(R)$ are sufficient to estimate the non-Gaussianity of $P[N(R)]$ and , hence, this fact avoids the need to compute higherorder moments. Third, the distance metric $l_{2}(R)$ provides a robust means to characterize the Gaussianity of the number distribution of general systems. Fourth, the decorrelation principle enabled us to show that a system's fluctuations become increasingly Gaussian or non-Gaussian, respectively, as the space dimensions increase.

The results of our analysis yield even wider applicability if the point configurations are mapped to two-phase media or random fields (by decorating each point with given shapes or kernels, respectively), which inherit the properties of the underlying point process. It is common practice to perform such mappings theoretically, numerically, and experimentally $[58,69,107,108]$. Thus, many of our results are expected to qualitatively extend to a broader class of materials than just many-particle systems. We note that a recent study of fluctuations in a 2D absorbing state lattice model finds number distributions with heavy left tails $\left[\gamma_{1}(R)<0\right]$, which are to be distinguished from the behavior of all our CLT models across dimensions with heavy right tails $\left[\gamma_{1}(R)>0\right][18]$.

A related but distinctly different study from the one considered in the present work concerns fluctuations when the window is centered on a point of the point process $[9,83]$. In future work, it will be interesting to carry out the analogous investigation of the corresponding skewness, excess kurtosis, and number distribution for such particlebased quantities for hyperuniform and nonhyperuniform models.

\section{ACKNOWLEDGMENTS}

This work was supported in part by the National Science Foundation under Grant No. DMR-1714722 and by the Princeton University Innovation Fund for New Ideas in the Natural Sciences. M. A. K. also acknowledges funding by the Volkswagenstiftung via the Experiment-Projekt Mecke. 


\section{APPENDIX: ASYMPTOTIC BEHAVIOR OF $l_{2}(R)$ FOR THE GAMMA DISTRIBUTION}

We prove that the large- $R$ scalings of the distance metric $l_{2}(R)$ and the skewness $\gamma_{1}(R)$ are the same for the gamma distribution. It is important to note that the asymptotic analysis is facilitated by transforming the random variable $N(R)$ to the standardized random variable $X \equiv(N(R)-\langle N(R)\rangle) / \sigma(R)$. In the large- $R$ limit, the discrete random variable tends to a continuous one, and hence the continuous-variable analog of the distance metric $l_{2}(R)$, defined Eq. (81), is given by

$$
l_{2}(R) \equiv\left[\int_{-\infty}^{\infty}\left|F_{G}(X)-F_{\Gamma}(X)\right|^{2} d X\right]^{1 / 2}
$$

where $F_{G}(X)$ and $F_{\Gamma}(X)$ are the cumulative distribution functions for the Gaussian and gamma distribution functions, respectively. Specifically,

$$
F_{G}(X)=\frac{1}{2}[1+\operatorname{erf}(X / \sqrt{2})]
$$

and

$$
F_{\Gamma}(X)=1-\frac{\Gamma(k, X \sqrt{k}+k)}{\Gamma(k)},
$$

where $\Gamma(s, y)=\int_{y}^{\infty} t^{s-1} \exp (-t) d t$ is the upper, incomplete gamma function.

Since the $(n+1)$ th cumulant of the gamma distribution tends to zero faster than its $n$th cumulant for $n \geq 3$, the greatest deviation of the gamma distribution from the normal one occurs in the vicinity of the origin, i.e., $X=0$, in the large- $k$ limit (see Fig. 8) and is thus dominated by the asymptotic behavior of the skewness. Thus, we require the Taylor series expansions of the distributions about $X=0$ :

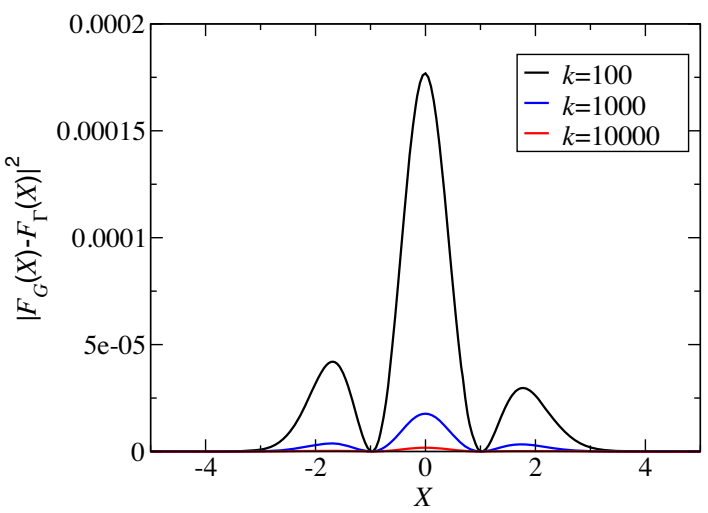

FIG. 8. Integrand of Eq. (A1) versus $X$ for select values of the shape parameter $k$.

$$
\begin{aligned}
F_{G}(X)= & \frac{1}{2}+\frac{1}{2} \sqrt{\frac{2}{\pi}} X-\frac{1}{12} \sqrt{\frac{2}{\pi}} X^{3}+\frac{1}{80} \sqrt{\frac{2}{\pi}} X^{5} \\
& -\frac{1}{672} \sqrt{\frac{2}{\pi}} X^{7}+\mathcal{O}\left(X^{9}\right), \\
F_{\Gamma}(X)= & a_{0}(k)+a_{1}(k) X+a_{2}(k) X^{2}+a_{3}(k) X^{3} \\
& +a_{4}(k) X^{4}+a_{5}(k) X^{5}+a_{6}(k) X^{6}+a_{7}(k) X^{7} \\
& +a_{8}(k) X^{8}+\mathcal{O}\left(X^{9}\right),
\end{aligned}
$$

where the coefficients $a_{m}(k)(m=1,2,3, \ldots)$ depend on the shape parameter $k$. We know these coefficients explicitly, but, for brevity, we do not mention them here. The corresponding large- $k$ asymptotic expansions of the first nine coefficients are

$$
\begin{gathered}
a_{0}(k)=\frac{1}{2}+\frac{1}{6} \sqrt{\frac{2}{k \pi}}+\mathcal{O}(1 / k), \\
a_{1}(k)=\frac{1}{2} \sqrt{\frac{2}{\pi}}+\mathcal{O}(1 / k),
\end{gathered}
$$

$$
\begin{aligned}
& a_{2}(k)=-\frac{1}{4} \sqrt{\frac{2}{k \pi}}+\mathcal{O}\left(1 / k^{3 / 2}\right), \\
& a_{3}(k)=-\frac{1}{12} \sqrt{\frac{2}{\pi}}+\mathcal{O}(1 / k),
\end{aligned}
$$

$$
\begin{gathered}
a_{4}(k)=\frac{5}{48} \sqrt{\frac{2}{k \pi}}+\mathcal{O}\left(1 / k^{3 / 2}\right), \\
a_{5}(k)=\frac{1}{80} \sqrt{\frac{2}{\pi}}+\mathcal{O}(1 / k), \\
a_{6}(k)=-\frac{7}{288} \sqrt{\frac{2}{k \pi}}+\mathcal{O}\left(1 / k^{3 / 2}\right), \\
a_{7}(k)=-\frac{1}{672} \sqrt{\frac{2}{\pi}}+\mathcal{O}(1 / k), \\
a_{8}(k)=\frac{1}{256} \sqrt{\frac{2}{k \pi}}+\mathcal{O}\left(1 / k^{3 / 2}\right) .
\end{gathered}
$$

We see that for odd $m$, the leading-order terms of $a_{m}$ are exactly the same as the coefficients multiplying $X^{m}$ in the series expansion (A4) for the standard normal distribution. Thus, combining Eqs. (A4) and (A5), and the aforementioned asymptotic expansions yields

$$
F_{\Gamma}(X)-F_{G}(X)=\frac{f(X)}{\sqrt{k}}+\mathcal{O}\left(\frac{1}{k^{3 / 2}}\right)
$$


where $f(X)$ is a function that is localized about the origin with the following corresponding Taylor series expansion:

$f(X)=\sqrt{\frac{2}{\pi}}\left[\frac{1}{6}-\frac{1}{4} X^{2}-\frac{5}{48} X^{4}-\frac{7}{288} X^{6}-\frac{1}{256} X^{8}+\mathcal{O}\left(X^{10}\right)\right]$.

Substitution of Eq. (A15) into Eq. (A1) gives the large- $k$ asymptotic expansion of the Gaussian distance metric, i.e.,

$$
l_{2}(R)=\frac{c}{\sqrt{k}}+\mathcal{O}\left(\frac{1}{k^{3 / 2}}\right)
$$

where the square of the constant $c$ is given by

$$
c^{2}=\int_{-\infty}^{\infty} f^{2}(X) d X
$$

Finally, since the skewness $\gamma_{1}(R)$ scales like $1 / \sqrt{k}$, we conclude that $l_{2}(R) \sim \gamma_{1}(R)$ for large values of $R$. Note that the vanishing of $l_{2}(R)$ for $R \rightarrow \infty$ implies a CLT for the gamma distribution as described in the text.

[1] P. Schofield, Wavelength-Dependent Fluctuations in Classical Fluids: I. The Long Wavelength Limit, Proc. Phys. Soc. 88, 149 (1966).

[2] D. J. Vezzetti, A New Derivation of Some Fluctuation Theorems in Statistical Mechanics, J. Math. Phys. (N.Y.) 16, 31 (1975).

[3] R. M. Ziff, On the Bulk Distribution Functions and Fluctuation Theorems, J. Math. Phys. (N.Y.) 18, 1825 (1977).

[4] F. Carmona and P. Delhaes, Effect of Density Fluctuations on the Physical Properties of a Disordered Carbon, J. Appl. Phys. 49, 618 (1978).

[5] J. P. Hansen and I. R. McDonald, Theory of Simple Liquids (Academic Press, New York, 1986).

[6] K. Jørgensen, J. H. Ipsen, O. G. Mouritsen, D. Bennett, and M. J. Zuckermann, The Effects of Density Fluctuations on the Partitioning of Foreign Molecules into Lipid Bilayers: Application to Anaesthetics and Insecticides, Biochim. Biophys. Acta 1067, 241 (1991).

[7] P. J. E. Peebles, Principles of Physical Cosmology (Princeton University Press, Princeton, NJ, 1993).

[8] P. M. Bleher, F. J. Dyson, and J. L. Lebowitz, NonGaussian Energy Level Statistics for Some Integrable Systems, Phys. Rev. Lett. 71, 3047 (1993).

[9] T. M. Truskett, S. Torquato, and P. G. Debenedetti, Density Fluctuations in Many-Body Systems, Phys. Rev. E 58, 7369 (1998).

[10] S. Torquato, Modeling of Physical Properties of Composite Materials, Int. J. Solids Struct. 37, 411 (2000).

[11] A. Gabrielli, M. Joyce, and F. S. Labini, Glass-like Universe: Real-Space Correlation Properties of Standard Cosmological Models, Phys. Rev. D 65, 083523 (2002).

[12] A. Wax, C. Yang, V. Backman, K. Badizadegan, C. W. Boone, R. R. Dasari, and M. S. Feld, Cellular Organization and Substructure Measured Using
Angle-Resolved Low-Coherence Interferometry, Biophys. J. 82, 2256 (2002).

[13] S. Torquato and F. H. Stillinger, Local Density Fluctuations, Hyperuniform Systems, and Order Metrics, Phys. Rev. E 68, 041113 (2003).

[14] A. C. Lavery, R. W. Schmitt, and T. K. Stanton, HighFrequency Acoustic Scattering from Turbulent Oceanic Microstructure: The Importance of Density Fluctuations, J. Acoust. Soc. Am. 114, 2685 (2003).

[15] M. A. Klatt and S. Torquato, Characterization of Maximally Random Jammed Sphere Packings. II. Correlation Functions and Density Fluctuations, Phys. Rev. E 94, 022152 (2016).

[16] S. Torquato, Hyperuniform States of Matter, Phys. Rep. 745, 1 (2018).

[17] F. L. Román, J. A. White, A. Gonzalez, and S. Velasco, Fluctuations in a Small Hard-Disk System: Implicit Finite Size Effects, J. Chem. Phys. 110, 9821 (1999).

[18] Y. Zheng, A. D. S. Parmar, and M. P. Ciamarra, Hidden Order Beyond Hyperuniformity in Critical Absorbing States, Phys. Rev. Lett. 126, 118003 (2021).

[19] A. Chremos and J. F. Douglas, Hidden Hyperuniformity in Soft Polymeric Materials, Phys. Rev. Lett. 121, 258002 (2018).

[20] Y. Zheng et al., Disordered Hyperuniformity in TwoDimensional Amorphous Silica, Sci. Adv. 6, eaba0826 (2020).

[21] A. Sheremet, R. Pierrat, and R. Carminati, Absorption of Scalar Waves in Correlated Disordered Media and Its Maximization Using Stealth Hyperuniformity, Phys. Rev. A 101, 053829 (2020).

[22] S. Wilken, R. E. Guerra, D. J. Pine, and P. M. Chaikin, Hyperuniform Structures Formed by Shearing Colloidal Suspensions, Phys. Rev. Lett. 125, 148001 (2020).

[23] Z. Ma, E. Lomba, and S. Torquato, Optimized Large Hyperuniform Binary Colloidal Suspensions in Two Dimensions, Phys. Rev. Lett. 125, 068002 (2020).

[24] G. Last and M. Penrose, Lectures on the Poisson Process, Institute of Mathematical Statistics Textbooks (Cambridge University Press, Cambridge, England, 2017).

[25] M. D. Penrose and J. E. Yukich, Limit Theory for Random Sequential Packing and Deposition, Ann. Appl. Probab. 12, 272 (2002).

[26] L. Heinrich, H. Schmidt, and V. Schmidt, Central Limit Theorems for Poisson Hyperplane Tessellations, Ann. Appl. Probab. 16, 919 (2006).

[27] T. Schreiber and J.E. Yukich, Limit Theorems for Geometric Functionals of Gibbs Point Processes, Ann. Inst. Henri Poincaré 49, 1158 (2013).

[28] B. Błaszczyszyn, D. Yogeshwaran, and J. E. Yukich, Limit Theory for Geometric Statistics of Point Processes Having Fast Decay of Correlations, Ann. Probab. 47, 835 (2019).

[29] S. Torquato, Random Heterogeneous Materials: Microstructure and Macroscopic Properties (Springer-Verlag, New York, 2002).

[30] U. Frisch, Wave Propagation in Random Media, in Probabilistic Methods in Applied Mathematics, 1st ed., edited by A. T. Bharucha-Reid (Academic Press, New York, 1968), Vol. 1, pp. 75-198. 
[31] P. Sheng, Introduction to Wave Scattering, Localization and Mesoscopic Phenomena (Academic Press, New York, 1995).

[32] L. Tsang, Scattering of Electromagnetic Waves, edited by J. A. Kong, Wiley Series in Remote Sensing (Wiley, Chichester, 2001).

[33] A. Cazé and J. C. Schotland, Diagrammatic and Asymptotic Approaches to the Origins of Radiative Transport Theory: Tutorial, J. Opt. Soc. Am. A 32, 1475 (2015).

[34] F. Zernike and J. A. Prins, Die Beugung von Röntgenstrahlen in Flüssigkeiten als Effekt der Molekülanordnung, Z. Phys. 41, 184 (1927).

[35] S. Torquato, Mean Nearest-Neighbor Distance in Random Packings of Hard d-Dimensional Spheres, Phys. Rev. Lett. 74, 2156 (1995).

[36] For equilibrium packings in $\mathbb{R}^{d}, S(0)$ is estimated from the fluctuation-compressibility relation $S(0)=\rho k_{B} T \kappa_{T}$, where $k_{B}$ is the Boltzmann constant, $T$ is the temperature, $\kappa_{T} \equiv \rho^{-1}(\partial \rho / \partial p)_{T}$ is the isothermal compressibility, and $p /\left(\rho k_{B} T\right)=G_{P}(\infty)$ is the reduced pressure.

[37] Analytic formula (6) is employed.

[38] S. Torquato, O. U. Uche, and F. H. Stillinger, Random Sequential Addition of Hard Spheres in High Euclidean Dimensions, Phys. Rev. E 74, 061308 (2006).

[39] G. Zhang and S. Torquato, Precise Algorithm to Generate Random Sequential Addition of Hard Hyperspheres at Saturation, Phys. Rev. E 88, 053312 (2013).

[40] Analytic formulas (70)-(73) are employed.

[41] B. Widom, Equation of State in the Neighborhood of the Critical Point, J. Chem. Phys. 43, 3898 (1965).

[42] L. P. Kadanoff, Scaling Laws for Ising Models near $T_{c}$, Physics 2, 263 (1966).

[43] M. E. Fisher, The Theory of Equilibrium Critical Phenomena, Rep. Prog. Phys. 30, 615 (1967).

[44] K. G. Wilson and J. Kogut, The Renormalization Group and the $\epsilon$ Expansion, Phys. Rep. 12, 75 (1974).

[45] H.E. Stanley, Introduction to Phase Transitions and Critical Phenomena (Oxford University Press, New York, 1987).

[46] J. J. Binney, N. J. Dowrick, A. J. Fisher, and M. E. J. Newman, The Theory of Critical Phenomena: An Introduction to the Renormalization Group (Oxford University Press, Oxford, England, 1992).

[47] V. A. Buryachenko, Micromechanics of Heterogeneous Materials (Springer, New York, 2007).

[48] J. Neyman and E. L. Scott, Statistical Approach to Problems of Cosmology, J. R. Stat. Soc. Ser. B 20, 1 (1958).

[49] S. N. Chiu, D. Stoyan, W. S. Kendall, and J. Mecke, Stochastic Geometry and Its Applications, 3rd ed. (Wiley, Chichester, 2013).

[50] J. Illian, A. Penttinen, H. Stoyan, and D. Stoyan, Statistical Analysis and Modelling of Spatial Point Patterns (John Wiley \& Sons, Chichester, 2008).

[51] D. Chandler, Introduction to Modern Statistical Mechanics (Oxford University Press, New York, 1987).

[52] J. P. Hansen and I. R. McDonald, Theory of Simple Liquids, 4th ed. (Academic Press, New York, 2013).

[53] W. X. Xu and H. S. Chen, Numerical Investigation of Effect of Particle Shape and Particle Size Distribution on Fresh Cement Paste Microstructure via Random
Sequential Packing of Dodecahedral Cement Particles, Comput. Struct. 114-115, 35 (2013).

[54] E. Roman and N. Majlis, Computer Simulation Model of the Structure of Ion Implanted Impurities in Semiconductors, Solid State Commun. 47, 259 (1983).

[55] J. Feder and I. Giaever, Adsorption of Ferritin, J. Colloid Interface Sci. 78, 144 (1980).

[56] P. J. Flory, Intramolecular Reaction between Neighboring Substituents of Vinyl Polymers, J. Am. Chem. Soc. 61, 1518 (1939).

[57] L. Finegold and J. T. Donnell, Maximum Density of Random Placing of Membrane-Particles, Nature (London) 278, 443 (1979).

[58] G. Hummer, S. Garde, A. E. García, A. Pohorille, and L. R. Pratt, An Information Theory Model of Hydrophobic Interactions, Proc. Natl. Acad. Sci. U.S.A. 93, 8951 (1996).

[59] J. C. Palmer, F. Martelli, Y. Liu, R. Car, A.Z. Panagiotopoulos, and P.G. Debenedetti, Metastable Liquid-Liquid Transition in a Molecular Model of Water, Nature (London) 510, 385 (2014).

[60] W. B. Russel, D. A. Saville, and W. R. Schowalter, Colloidal Dispersions (Cambridge University Press, Cambridge, England, 1989).

[61] M. A. Klatt, J. Kim, and S. Torquato, Cloaking the Underlying Long-Range Order of Randomly Perturbed Lattices, Phys. Rev. E 101, 032118 (2020).

[62] T. R. Welberry, G. H. Miller, and C.E. Carroll, Paracrystals and Growth-Disorder Models, Acta Crystallogr. Sect. A 36, 921 (1980).

[63] W. J. Stroud and R. P. Millane, Cylindrically Averaged Diffraction by Distorted Lattices, Proc. R. Soc. A 452, 151 (1996).

[64] N. W. Ashcroft and D. N. Mermin, Solid State Physics (Thomson Learning, Toronto, 1976).

[65] P. M. Chaikin and T. C. Lubensky, Principles of Condensed Matter Physics (Cambridge University Press, New York, 1995).

[66] J. Kim and S. Torquato, Effect of Imperfections on the Hyperuniformity of Many-Body Systems, Phys. Rev. B 97, 054105 (2018).

[67] O. Christogeorgos, H. Zhang, Q. Cheng, and Y. Hao, Extraordinary Directive Emission and Scanning from an Array of Radiation Sources with Hyperuniform Disorder, Phys. Rev. Applied 15, 014062 (2021).

[68] R. Degl'Innocenti, Y. D. Shah, L. Masini, A. Ronzani, A. Pitanti, Y. Ren, D. S. Jessop, A. Tredicucci, H. E. Beere, and D. A. Ritchie, Hyperuniform Disordered Terahertz Quantum Cascade Laser, Sci. Rep. 6, 19325 (2016).

[69] M. Florescu, S. Torquato, and P. J. Steinhardt, Designer Disordered Materials with Large Complete Photonic Band Gaps, Proc. Natl. Acad. Sci. U.S.A. 106, 20658 (2009).

[70] J.H. Conway and N. J. A. Sloane, Sphere Packings, Lattices and Groups (Springer-Verlag, New York, 1998).

[71] It is interesting to note that the determination of $P[N(R)]$ in models of liquid water is central to understanding cavity formation in such systems. In particular, finding the radius at which the distribution is approximately normal provides a Gaussian-based field theory for solvation; see Ref. [58] and references therein. 
[72] S. Torquato and F. H. Stillinger, New Conjectural Lower Bounds on the Optimal Density of Sphere Packings, Exp. Math. 15, 307 (2006).

[73] A. Scardicchio, F. H. Stillinger, and S. Torquato, Estimates of the Optimal Density of Sphere Packings in High Dimensions, J. Math. Phys. (N.Y.) 49, 043301 (2008).

[74] M. Skoge, A. Donev, F. H. Stillinger, and S. Torquato, Packing Hyperspheres in High-Dimensional Euclidean Spaces, Phys. Rev. E 74, 041127 (2006).

[75] S. Torquato and F. H. Stillinger, Exactly Solvable Disordered Sphere-Packing Model in Arbitrary-Dimensional Euclidean Spaces, Phys. Rev. E 73, 031106 (2006).

[76] S. Torquato, A. Scardicchio, and C. E. Zachary, Point Processes in Arbitrary Dimension from Fermionic Gases, Random Matrix Theory, and Number Theory, J. Stat. Mech. (2008) P11019.

[77] C. E. Zachary, F. H. Stillinger, and S. Torquato, GaussianCore Model Phase Diagram and Pair Correlations in High Euclidean Dimensions, J. Chem. Phys. 128, 224505 (2008).

[78] S. Torquato, Reformulation of the Covering and Quantizer Problems as Ground States of Interacting Particles, Phys. Rev. E 82, 056109 (2010).

[79] S. Torquato, Perspective: Basic Understanding of Condensed Phases of Matter via Packing Models, J. Chem. Phys. 149, 020901 (2018).

[80] K. Pearson, IX. Mathematical Contributions to the Theory of Evolution. XIX. Second Supplement to a Memoir on Skew Variation, Phil. Trans. R. Soc. A 216, 429 (1916).

[81] See Supplemental Material at http://link.aps.org/ supplemental/10.1103/PhysRevX.11.021028 for additional plots and derivations.

[82] D. S. Broca, Cumulant-Moment Relations through Determinants, Int. J. Math. Educ. Sci. Technol. 35, 917 (2004).

[83] S. Torquato, B. Lu, and J. Rubinstein, Nearest-Neighbor Distribution Functions in Many-Body Systems, Phys. Rev. A 41, 2059 (1990).

[84] A. B. Hopkins, F. H. Stillinger, and S. Torquato, Densest Local Sphere-Packing Diversity: General Concepts and Application to Two Dimensions, Phys. Rev. E 81, 041305 (2010).

[85] A. B. Hopkins, F. H. Stillinger, and S. Torquato, Densest Local Sphere-Packing Diversity: Application to Three Dimensions, Phys. Rev. E 83, 011304 (2011).

[86] G. Zhang, F. H. Stillinger, and S. Torquato, Can Exotic Disordered "Stealthy" Particle Configurations Tolerate Arbitrarily Large Holes?, Soft Matter 13, 6197 (2017).

[87] D. Levine and P. J. Steinhardt, Quasicrystals: A New Class of Ordered Structures, Phys. Rev. Lett. 53, 2477 (1984).

[88] S. Ghosh and J. L. Lebowitz, Generalized Stealthy Hyperuniform Processes: Maximal Rigidity and the Bounded Holes Conjecture, Commun. Math. Phys. 363, 97 (2018).

[89] Thus, for any system of hard spheres of diameter $D$ with packing fraction $\phi=\rho v_{1}(D / 2)$, we see that fixing $\rho$ to unity implies that $D$ changes with the packing fraction.

[90] C. E. Zachary and S. Torquato, Hyperuniformity in Point Patterns and Two-Phase Heterogeneous Media, J. Stat. Mech. (2009) P12015.

[91] S. Torquato, Structural Characterization of ManyParticle Systems on Approach to Hyperuniform States, arXiv:2103.14989.
[92] A. Reńyi, On a One-Dimensional Random Space-Filling Problem, Publ. Math. Res. Inst. Hung. Acad. Sci. 3, 109 (1958).

[93] B. Widom, Random Sequential Addition of Hard Spheres to a Volume, J. Chem. Phys. 44, 3888 (1966).

[94] J. Feder, Random Sequential Adsorption, J. Theor. Biol. 87, 237 (1980).

[95] D. W. Cooper, Random-Sequential-Packing Simulations in Three Dimensions for Spheres, Phys. Rev. A 38, 522 (1988).

[96] B. Bonnier, D. Boyer, and P. Viot, Pair Correlation Function in Random Sequential Adsorption Processes, J. Phys. A 27, 3671 (1994).

[97] M. A. Klatt, J. Lovrić, D. Chen, S. C. Kapfer, F. M. Schaller, P. W. A. Schönhöfer, B. S. Gardiner, A.-S. Smith, G. E. Schröder-Turk, and S. Torquato, Universal Hidden Order in Amorphous Cellular Geometries, Nat. Commun. 10, 811 (2019).

[98] R. Schneider and W. Weil, Stochastic and Integral Geometry (Probability and Its Applications) (Springer, Berlin, Germany, 2008).

[99] C. F. Gauss, Besprechung des Buchs von L. A. Seeber: Untersuchungen über die Eigenschaften der Positiven Ternären Quadratischen Formen, Göttingsche Gelehrte Anzeigen (1831); See also J. Reine, Angew. Math. 20, 312 (1840).

[100] P. Sarnak and A. Strömbergsson, Minima of Epstein's Zeta Function and Heights of Flat Tori, Inventiones Mathematicae 165, 115 (2006).

[101] Y. Fan, J. K. Percus, D. K. Stillinger, and F. H. Stillinger, Constraints on Collective Density Variables: One Dimension, Phys. Rev. A 44, 2394 (1991).

[102] O. U. Uche, F. H. Stillinger, and S. Torquato, Constraints on Collective Density Variables: Two Dimensions, Phys. Rev. E 70, 046122 (2004).

[103] O. U. Uche, S. Torquato, and F. H. Stillinger, Collective Coordinates Control of Density Distributions, Phys. Rev. E 74, 031104 (2006).

[104] R. D. Batten, F. H. Stillinger, and S. Torquato, Classical Disordered Ground States: Super-Ideal Gases, and Stealth and Equi-luminous Materials, J. Appl. Phys. 104, 033504 (2008).

[105] S. Torquato, G. Zhang, and F. H. Stillinger, Ensemble Theory for Stealthy Hyperuniform Disordered Ground States, Phys. Rev. X 5, 021020 (2015).

[106] G. Zhang, F. Stillinger, and S. Torquato, Ground States of Stealthy Hyperuniform Potentials: I. Entropically Favored Configurations, Phys. Rev. E 92, 022119 (2015).

[107] O. Leseur, R. Pierrat, and R. Carminati, High-Density Hyperuniform Materials Can Be Transparent, Optica 3, 763 (2016).

[108] S. Torquato, Hyperuniformity and Its Generalizations, Phys. Rev. E 94, 022122 (2016).

[109] N. Henze, Invariant Tests for Multivariate Normality: A Critical Review, Statistical papers 43, 467 (2002).

[110] S. Kullback and R.A. Leibler, On Information and Sufficiency, Ann. Math. Stat. 22, 79 (1951).

[111] H. Wang, F. H. Stillinger, and S. Torquato, Sensitivity of Pair Statistics on Pair Potentials in Many-Body Systems, J. Chem. Phys. 153, 124106 (2020). 
[112] B. Klemens, Modeling with Data: Tools and Techniques for Scientific Computing (Princeton University Press, Princeton, NJ, 2009), online Appendix M.

[113] We expect this data cut to be conservative because the $l_{2}$ distance metric contains information of all moments and higher moments are generally less numerically robust. By visual inspection, we found a similar range of radii for which we have reliable estimates for $\gamma_{1}, \gamma_{2}$, and $l_{2}$.

[114] S. Torquato, J. Kim, and M. A. Klatt, Supplementary dataset: Local Number Fluctuations in Hyperuniform and Nonhyperuniform Systems: Higher-Order Moments and Distribution Functions, Zenodo, https://doi.org/ 10.5281/zenodo.4635977.

[115] In a less-detailed fluctuation study of the ideal gas, equilibrium hard spheres, and MRJ sphere packings, Klatt and Torquato [15] consistently found that the convergence of the number distributions to a Gaussian distribution was slowest for the Poisson distribution and fastest for hyperuniform MRJ sphere packings. 